\title{
Can Medical Herbs Stimulate Regeneration or Neuroprotection and Treat Neuropathic Pain in Chemotherapy-Induced Peripheral Neuropathy?
}

\author{
Sven Schröder, ${ }^{1,2}$ Kathrin Beckmann, ${ }^{1}$ Giovanna Franconi, ${ }^{3}$ Gesa Meyer-Hamme, ${ }^{1}$ \\ Thomas Friedemann, ${ }^{1}$ Henry Johannes Greten, ${ }^{2}$ Matthias Rostock, ${ }^{4}$ and Thomas Efferth ${ }^{5}$ \\ ${ }^{1}$ HanseMerkur Center for Traditional Chinese Medicine at the University Medical Center Hamburg-Eppendorf, \\ Martinistraße 52, 20246 Hamburg, Germany \\ ${ }^{2}$ ICBAS, University of Porto, Rua de Jorge Viterbo Ferreira No. 228, 4050-313 Porto, Portugal \\ ${ }^{3}$ Department of Systems Medicine, Tor Vergata University, 00133 Rome, Italy \\ ${ }^{4}$ Hubertus Wald Tumorzentrum, University Cancer Center Hamburg, University Medical Center Hamburg-Eppendorf, \\ Martinistraße 52, 20246 Hamburg, Germany \\ ${ }^{5}$ Department of Pharmaceutical Biology, Institute of Pharmacy and Biochemistry, Johannes Gutenberg University, \\ Staudinger Weg 5, 55128 Mainz, Germany
}

Correspondence should be addressed to Sven Schröder; schroeder@tcm-am-uke.de

Received 30 April 2013; Accepted 5 June 2013

Academic Editor: Yoshiharu Motoo

Copyright (C) 2013 Sven Schröder et al. This is an open access article distributed under the Creative Commons Attribution License, which permits unrestricted use, distribution, and reproduction in any medium, provided the original work is properly cited.

\begin{abstract}
Chemotherapy-induced neuropathy (CIPN) has a relevant impact on the quality of life of cancer patients. There are no curative conventional treatments, so further options have to be investigated. We conducted a systematic review in English and Chinese language databases to illuminate the role of medical herbs. 26 relevant studies on 5 single herbs, one extract, one receptoragonist, and 8 combinations of herbs were identified focusing on the single herbs Acorus calamus rhizoma, Cannabis sativa fructus, Chamomilla matricaria, Ginkgo biloba, Salvia officinalis, Sweet bee venom, Fritillaria cirrhosae bulbus, and the herbal combinations Bu Yang Huan Wu, modified Bu Yang Huan Wu plus Liuwei Di Huang, modified Chai Hu Long Gu Mu Li Wan, Geranii herba plus Aconiti lateralis praeparata radix, Niu Che Sen Qi Wan (Goshajinkigan), Gui Zhi Jia Shu Fu Tang (Keishikajutsubuto), Huang Qi Wu Wu Tang (Ogikeishigomotsuto), and Shao Yao Gan Cao Tang (Shakuyakukanzoto). The knowledge of mechanism of action is still limited, the quality of clinical trials needs further improvement, and studies have not yielded enough evidence to establish a standard practice, but a lot of promising substances have been identified. While CIPN has multiple mechanisms of neuronal degeneration, a combination of herbs or substances might deal with multiple targets for the aim of neuroprotection or neuroregeneration in CIPN.
\end{abstract}

\section{Introduction}

Several chemotherapeutic drugs are known to be neurotoxic and can lead to chemotherapy-induced peripheral neuropathy (CIPN). It is one of the main dose-limiting toxicities in oncologic treatments and a potential reason to terminate or suspend chemotherapy, in some cases leading to disease progression [1]. CIPN involves damage to the peripheral nervous system and can produce severe neuropathic pain $[2,3]$, sensory deficits, or gait impairment [4] and can severely decrease the patient's quality of life [5]. Sensory symptoms usually develop before motor symptoms, because motor neurons are more myelinated [6,7]. Distal parts of the axons are the first affected, so sensory symptoms typically start symmetrically and bilaterally from the tips of the toes and fingers and progress proximally in a "stocking-glove" distribution [8]. The incidence of CIPN can reach levels of up to $92 \%$ [9]. The major groups of drugs that induce CIPN include the antitubulins (paclitaxel, docetaxel, ixabepilone, and vincristine), platinum analogs (cisplatin, carboplatin, and oxaliplatin), and the proteasome inhibitors bortezomib and thalidomide [1]. Patients with a preexisting other cause of 
peripheral neuropathy develop more severe and persistent CIPN [10-12].

The development of CIPN is usually dependent on the cumulative dose and symptoms may progressively aggravate [10]. After sustaining therapy symptoms are usually reversible, but in some cases they may be irreversible $[10,13]$ and sometimes even progress after stopping medication, especially after treatment with vinca alkaloids (e.g., vincristine), platinum analogues (e.g., cisplatin and oxaliplatin), and taxanes (e.g., paclitaxel) $[14,15]$. Mostly the sensory nerve cell bodies of the dorsal root ganglia are affected, or the afferent and efferent distal peripheral axons are damaged [16].

Some differences concerning the mechanisms of CIPN are described for different therapeutic drugs.

In platinum compounds the dorsal root ganglia are the main parts of the nervous systems that are injured [17] and apoptosis is induced via structural alterations of DNA and of cell-cycle kinetics [18], triggered by oxidative stress and mitochondrial dysfunction but possibly downregulated by a reduction of enzymes like p53 [19-22].

The taxanes paxlitaxel and docetaxel are antitubulins and mainly damage the soma of the sensory neurons and the nerve axons. This is induced by an interference with the axonal transport, which is caused by enhancement of microtubule polymerization [23]. Microglial activation in the spinal cord and high concentration in the dorsal root ganglia tissue induce CIPN [24]. Like platinum compounds, taxanes can damage dorsal root ganglia. This is induced by macrophage activation in the dorsal root ganglia but occurs also in the peripheral nerve [24]. Paclitaxel induces a massive polar reconfiguration of axonal microtubules and an impairment of organelle transport [25].

Vinca alkaloids (e.g., vincristine) prevent tubulin polymerization from soluble dimers into microtubules [26]. By affecting the tubulin dimers loss of axonal microtubules and alterations in their length, arrangement and orientation are produced $[27,28]$. This alters the neuronal cytoskeleton, leading to abnormalities in axonal transport and axonal degeneration $[28,29]$. Decreasing affinity for tubulin of the vinca alkaloids explains the different neurotoxicity profiles and the severity of CIPN [30].

Epothilones (e.g., ixabepilone) are like the taxanes antitubulins, so there might be similar mechanisms of peripheral neurotoxicity. They damage the ganglion soma cells and peripheral axons through disruption of microtubules of the mitotic spindle and interfere with the axonal transport in the neurons [31] and can also induce polymerization of tubulin dimers in microtubules. Additionally they stabilize preformed microtubules against conditions favouring depolymerization $[32,33]$.

Bortezomib might induce pathological changes in Schwann cells and myelin, axonal degeneration, and dorsal root ganglia neuron changes [34, 35], as well as chromatolysis of dorsal root ganglial neurons. It causes cytoplasmic accumulation of eosinophilic material [36] and interferes with the transcription, nuclear processing and transport, as well as with the cytoplasmic translation of messenger RNAs in dorsal root ganglions [37]. Mitochondrial and endoplasmic reticulum-mediated calcium dysregulation plays an important part $[38,39]$. The activation of the mitochondria-based apoptotic pathway or inhibition of the transcription of the nerve growth factor by interference with the nuclear factor$\kappa \mathrm{B}$ pathway can lead to disarrangement of the neurotrophin network [39, 40]. Bortezomib can induce changes in all major primary afferent fibres [41].

The structure of thalidomide is characterized by a 3substituted glutarimide ring and a ph-thalimide ring, which are sensitive to enzymatic or nonenzymatic hydrolysis [42], but despite several studies it has not been possible to identify the responsible enzymes for the production of neurotoxic metabolites, so its mechanism of peripheral neurotoxicity is still elusive [43].

Multiple drugs have been tested, mainly in animal studies, for their putative neuroprotective activity in CIPN. A few components which can protect different tissues from toxic agents were clinically tested, showing conflicting results. No conclusive reports confirming their effectiveness have been provided $[44,45]$ and a reduction of anticancer activity was suspected [46]. Several neurotrophins were tested but there was no evidence of neuroprotection [47-50]. Erythropoietin, a multifunctional trophic factor, showed promising results in preclinical studies $[51,52]$ but has relevant safety problems [53].

Antioxidants have been tested as neuroprotectants [5459], but no conclusive evidence of neuroprotection has yet been found.

Neuropathic pain is conventionally treated by antiepileptic and tricyclic antidepressant drugs, but these drugs are ineffective for treatment of decreased sensation and cannot induce neuroprotection or neuroregeneration. Other compounds with different mechanisms (e.g., acetyl-L-carnitine, glutamate carboxypeptidase II inhibitors, calpain inhibitors, a solution of calcium and magnesium) have now been investigated in preclinical stages with unknown value for clinical routine [59-63].

Thus specific and effective curative treatments for CIPN are lacking, especially those meant for enhancing neuroregeneration or neuroprotection [64-67], and the evaluation of further treatment options is of great importance. While the effectiveness of herbal treatment is not well known yet, this review was done to illuminate the actual and potential future role of herbal treatment in CIPN.

\section{Methods}

To review the existing clinical and experimental studies of herbal treatment in CIPN, a systematic literature search was performed from the databases from inception up until January 2013 using MEDLINE, Google Scholar, Cochrane Database, CINHAL (Cumulative Index to Nursing and Allied Health Literature), CNKI (China National Knowledge Infrastructure), and Wanfang Med Online and ISI Proceedings for conference abstracts. The keywords searched were as follows:

(Chinese herbs or herbs or plants or Chinese medicine as MeSH term) AND (neuropathy or chemotherapy). The CINHAL, CNKI, and Wanfang Med Online Databases did 
not allow logical searches with AND, so we used simple combinations of the search words. Historical searches of reference lists of relevant articles were also undertaken.

To be included in our review a study had to focus on the topic CIPN and neuropathy in human and animal models irrespective of design. Papers with at least an English abstract were included. Study selection was performed by two reviewers (SSch and $\mathrm{KB}$ ) with disagreement resolved by discussion and adjudication.

Listed articles concerning diseases other than CIPN and herbal treatment were excluded, while animal products used in the tradition of herbal medicine were included.

\section{Results}

A total of 3474 (1477 in English and 1997 in Chinese databases) articles were retrieved by way of electronic searches and examination of reference lists of clinical and review articles. After screening titles and/or abstracts, 3376 articles were excluded because either the focus was on an intervention other than CIPN and herbal treatment or they were duplicated studies or not relevant. From a total of 98 articles which were retrieved for detailed evaluation, 15 were included in the review, focusing on 5 single herbs, one extract, one receptor agonist, and 8 combinations of herbs. For a summary of the experimental and clinical studies see Table 1 .

\subsection{Single Herbs or Single Herbal Compounds}

3.1.1. Acorus calamus rhizoma. This herb (family: Araceae) is traditionally used in the treatment and management of pain and severe inflammatory in Ayurveda. It is commonly used to relieve the muscle, joint, vascular and nerve injury associated with severe inflammatory and neuropathic pain [68]. In a rat model a hydroalcoholic extract of Acorus calamus rhizoma has been shown to exert beneficial effect on neuropathic pain induced by tibial and sural nerve transection [69]. In a further study Acorus calamus rhizoma extract attenuated sciatic nerve chronic constriction injury and induced ameliorated behavioral (hyperalgesia and allodynia), biochemical (superoxide anion, myeloperoxidase, and total calcium), and histopathological (axonal degeneration) changes [68]. Another study investigated the protective effect of Acorus calamus rhizoma extract in vincristine-induced painful neuropathy. Hydroalcoholic extracts of Acorus calamus rhizoma attenuated vincristine-induced behavioral and biochemical changes to an extent comparable to pregabalin (positive control) and attenuated vincristine-induced painful neuropathy, which probably may be attributed to its multiple effects including antioxidative, anti-inflammatory, and calcium inhibitory activity [70].

3.1.2. Cannabis sativa. Two structurally distinct cannabinoid CB2 agonists-the aminoalkylindole (R,S)-AM1241 $((\mathrm{R}, \mathrm{S})$-(2-iodo-5-nitrophenyl)- [1-((1-methyl-piperidin-2yl)methyl)-1H-indol-3-yl]-methanone) and the cannabilactone AM1714 (1,9-dihydroxy-3-(1',1'-dimethylheptyl)-6Hbenzo[c]chromene-6-one))-had been tested for their dose related suppression of established paclitaxel-evoked mechanical allodynia in a rat model. (R)-AM1241, but not (S)AM1241, suppressed paclitaxel evoked mechanical allodynia, and AM1714 induced a modest antinociceptive effect. So the authors suggested that cannabinoid CB2 receptors may be important therapeutic targets for the treatment of chemotherapy-evoked neuropathy [71]. Cannabis sativa or Cannabis extracts have not been clinically explicitly tested for CIPN but for other clinical conditions like neuropathic pain in HIV [72]. Multiple clinical trials examined the effect on neuropathic pain and found positive effects on central and peripheral neuropathic pain with different forms of application [73-82].

3.1.3. Matricaria chamomilla. This is a commonly used herb in western as well as in eastern phytopharmacological tradition. Flavonoids from Matricaria chamomilla seem to have an antispasmodic effect and main components such as $\alpha$-bisabolol or chamazulene have anti-inflammatory effects [83]. En-In-Dicycloether has both antispasmodic and antiinflammatory effects together [84]. In a mouse model it was shown that Matricaria chamomilla extract-treated mice had a significant reduction of cisplatin-induced peripheral pain [85]. Matricaria chamomilla hydroalcoholic extract was able to decrease cisplatin-induced pain and inflammation better than morphine [85].

3.1.4. Ginkgo biloba. The popular herb from the maidenhair tree that has shown some promising effects as an neuroprotectant. The most unique components of the extracts are the terpene trilactones, that is, ginkgolides and bilobalide [109]. In vitro and ex vivo studies indicate that bilobalide has multiple mechanisms of action that may be associated with neuroprotection, including its preservation of mitochondrial ATP synthesis, its inhibition of apoptotic damage induced by staurosporine or by serum-free medium, its suppression of hypoxia-induced membrane deterioration in the brain, and its action in increasing the expression of the mitochondrial DNA-encoded COX III subunit of cytochrome C oxidase and the ND1 subunit of NADH dehydrogenase [110]. Because multiple modes of action may apply to bilobalide, it could be useful in developing therapy for neurodegeneration [109111]. Oztürk et al. investigated Ginkgo biloba alcoholic extract in cisplatin-induced peripheral neuropathy in mice [86]. Development of neuropathy was evaluated with changes in sensory nerve conduction velocity (NCV) and Ginkgo biloba extract prevented reduction in NCV. In another study a Ginkgo biloba extract prevented some functional and morphological deteriorations induced by cisplatin, antagonizing the decrease in the number of migrating cells and in the length of outgrowing axons [86]. Marshall et al. investigated retrospectively 17 patients with colorectal cancer who received oxaliplatin along with Ginkgo biloba extract, but no specification of the extraction method was provided in the published abstract. The researchers found that 11 of the 17 patients developed a grade 1 peripheral neuropathy (PN) after the first cycle of oxaliplatin. Five of six patients who received Ginkgo biloba after the second cycle of oxaliplatin 


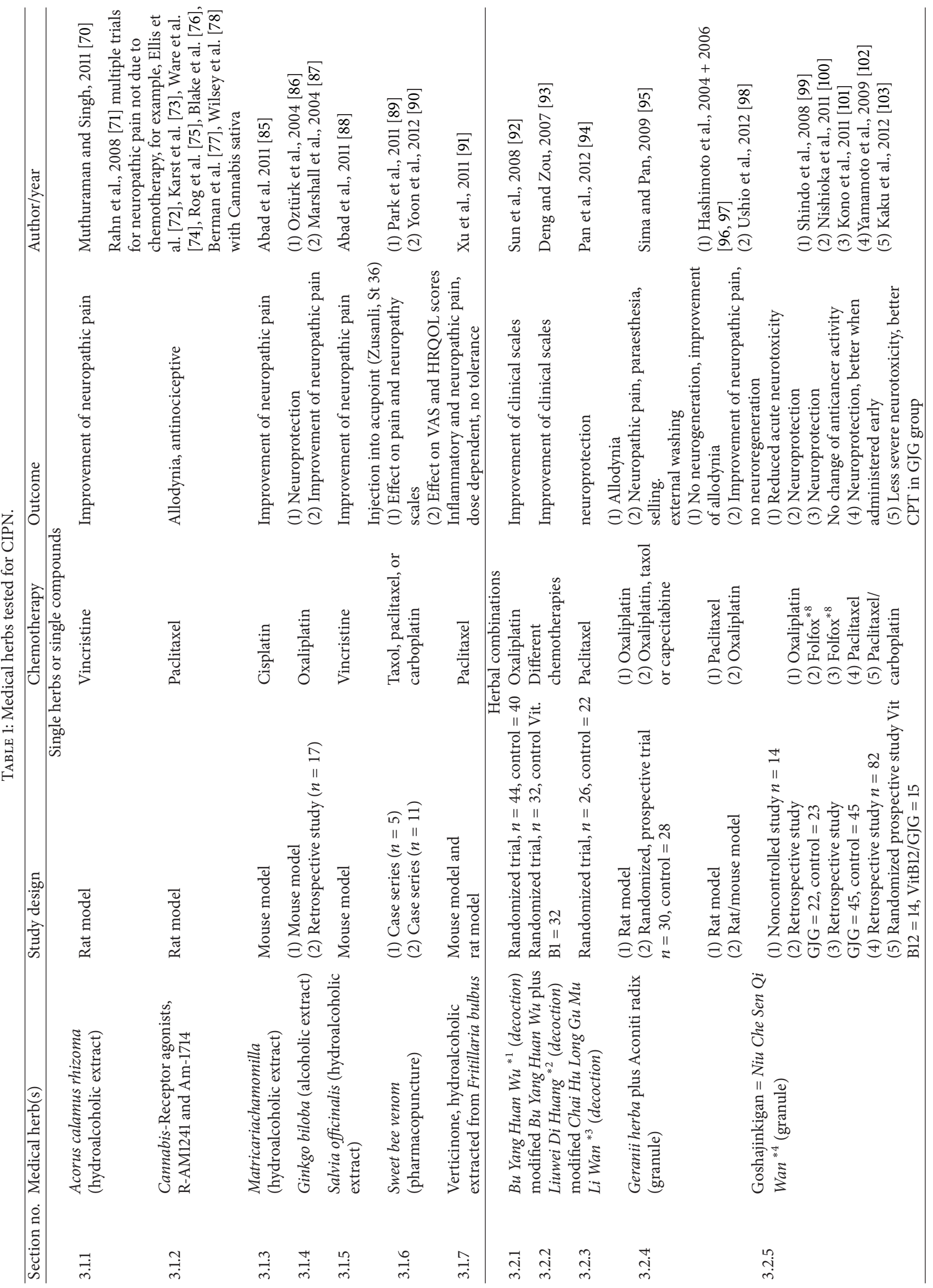




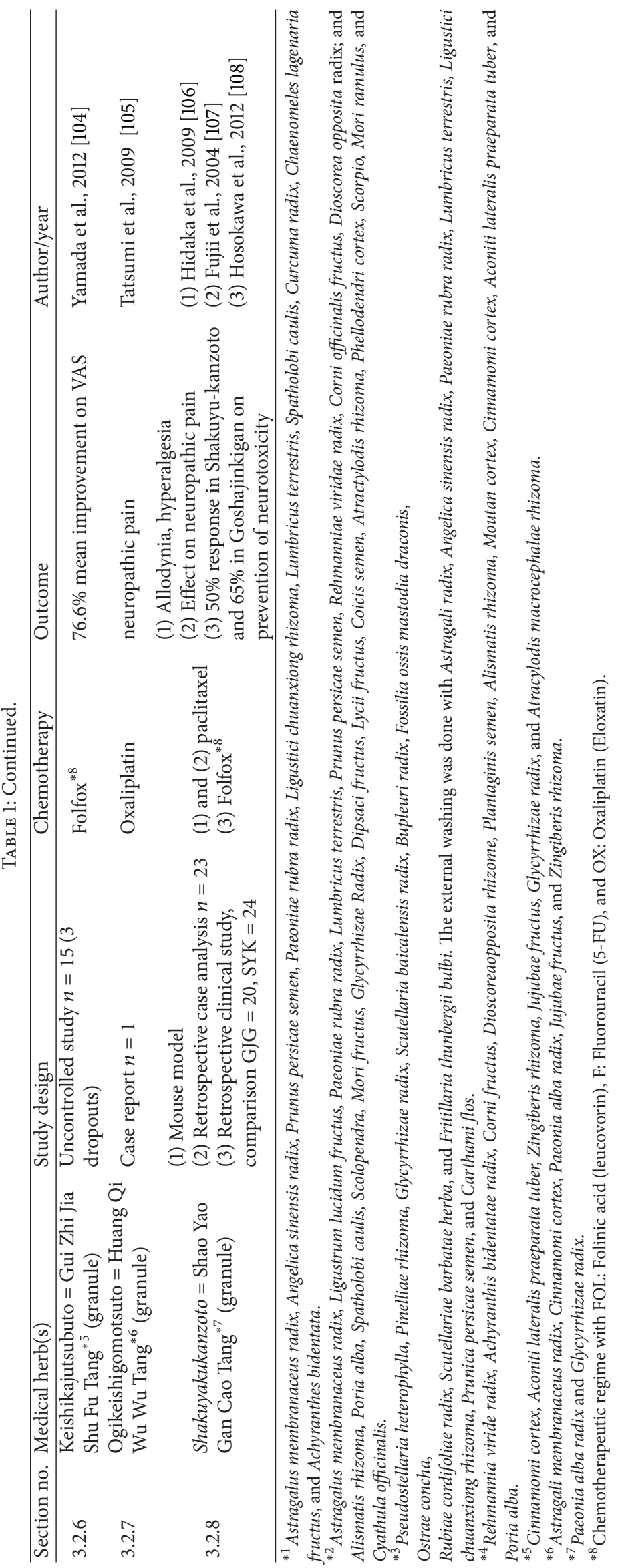


reported decreased intensity and duration of sensory PN. No Ginkgo biloba related side effects have been observed. The data suggested that Ginkgo biloba extract appears to attenuate the intensity and duration of acute dysesthesias caused by oxaliplatin and may yield synergistic antitumor activity [87].

3.1.5. Salvia officinalis. Salvia species and their isolated constituents possess significant antioxidant activity in enzymedependent and enzyme-independent systems [112-115]. The flavonoid apigenin, for example, has been shown to protect neurons against $\mathrm{A} \beta$-induced toxicity [116]. In addition to antioxidant activity, many salvia species and their isolated constituents showed anti-inflammatory properties [117, 118]. Salvia officinalis extract can have anti-inflammatory and also antinociceptive effects on chemical behavioral models of nociception in mice that involve an opioid mechanism [119]. An animal study showed the effects of the Salvia officinalis hydroalcoholic extract on vincristine-induced $\mathrm{PN}$ in mice in comparison with morphine with a decrease of pain response, suggesting that Salvia officinalis extract could be useful in the treatment of vincristine-induced peripheral neuropathic pain [88].

3.1.6. Sweet bee venom. The venom of honey bees with its active peptide Melittin has been tested for injection into the acupuncture point Zusanli (ST 36) for its effect on CIPN in animal models. It showed to alleviate thermal hyperalgesia and mechanical allodynia. The results indicated an association with the activation of the LC noradrenergic system and with a reduction in spinal pNR1 [120, 121].

In a first case series this was tested in 5 patients in a 1-week course of treatment, which showed no side effects and gave evidence of clinical improvement [89]. Another prospective case series of this procedure analyzed the clinical observations made on 11 CIPN patients treated with Sweet bee venom. A total of 11 eligible consecutive CIPN patients were treated for 3 weeks and observed for another 3 weeks. A significant intraindividual improvement was found for pain and neuropathy scales [90].

3.1.7. Verticinone from Fritillaria bulbus. Verticinone, an isosteroidal alkaloid isolated from Fritillaria bulbus, was evaluated in mice for its analgesic activities in murine models of inflammatory and neuropathic pain. It was shown that oral administration of hydroalcoholic extracted verticinone could significantly inhibit acetic acid-induced writhing response in a dose-dependent manner superior to acetylsalicyl acid. In the rat model of paclitaxel induced neuropathic pain, in contrast to the declined analgesic effect of morphine after repeated administration with the same dose, a relatively constant analgesic effect of verticinone was observed, so verticinone is expected to become a potentially novel sedativeanalgesic agent without producing tolerance [91].

\subsection{Herbal Combinations}

3.2.1. Bu Yang Huan Wu (Chin.) = Tonify the Yang to Restore Five-Tenths Decoction (Engl.). Bu Yang Huan Wu is a classical combination of Chinese herbs, first mentioned in Wang Qing-Ren's Yi Lin Gai Cuo (Correcting the Errors in the Field of Medicine) published in 1830 [122]. This recipe contains Astragalus membranaceus radix, Angelica sinensis radix, Prunus persicae semen, Paeoniae rubra radix, Ligustici chuanxiong rhizoma, Lumbricus terrestris, Spatholobi caulis, Curcuma radix, Chaenomeles lagenaria fructus, and Achyranthes bidentatae radix.

The decoction has been used in a randomized Chinese study of 84 patients (treatment group $n=44$, control group $n=40$ ) after the treatment of oxaliplatin and showed reduced development of CIPN in the treatment group tested by standardized clinical tests [92].

3.2.2. Modified Bu Yang Huan Wu (Chin.) = Tonify the Yang to Restore Five-Tenths Decoction (Engl.) plus Liuwei Di Huang (Chin.) = Rokumijiogan (Jap.) = Pilula Rehmannia Sex Saporum (Lat.) = Six Ingredients Pill with Rehmannia (Engl.). In another randomized Chinese study a modified combination of two standard recipes Bu Yang Huan Wu and Liuwei Di Huang was tested as a decoction. Liuwei Di Huang was first described in the Yozheng Zhijue [123]. The mixture of both recipes contains Astragalus membranaceus radix, Ligustrum lucidum fructus, Paeoniae rubra radix, Lumbricus terrestris, Prunus persicae semen, Rehmanniae viride radix, Corni officinalis fructus, Dioscorea opposita radix; and Alismatis rhizoma, Poria alba, Spatholobi caulis, Scolopendra, Mori fructus, Glycyrrhizae radix, Dipsaci fructus, Lycii fructus, Coicis semen, Atractylodis rhizoma, Phellodendri cortex, Scorpio, Mori ramulus, and Cyathula officinalis.

The remaining dregs of decoction were additionally used for washing the lower extremities. The treatment was used on 32 patients with existing CIPN following different chemotherapies and compared with 32 patients who were treated orally with vitamin B1 2500 ug plus by intramuscular injection with vitamin B1 $100 \mathrm{mg}$. Herbal treatment was found to be significantly more effective to vitamin treatment $(P<$ 0.05) [93].

3.2.3. Modified Chai Hu Long Gu Mu Li Wan (Chin.) = Modified Saikokaryukotsuboreito (Jap.) = Modified Formula bupleurum cum ostrea et Fossilia ossis (Lat.) = Modified Bupleurum, Dragon Bone, and Oyster Shell Formula (Engl.). Chai Hu Long Gu Mu Li Wan is a traditional recipe derived from the Shang Han Lun [124]. A modification of this recipe was used in a Chinese randomized trial in which 48 patients with ovarian cancer were examined parallel to chemotherapy with placitaxel. They were divided into a treatment group with paclitaxel alone and a treatment group with paclitaxel plus a combination of oral Chinese herbal decoction treatment and external washing of the feet with Chinese herbs.

The oral combination of herbs consists of Pseudostellaria heterophylla, Pinelliae rhizoma, Glycyrrhizae radix, Scutellaria baicalensis radix, Bupleuri radix, Fossilia ossis mastodi, Ostreae concha, Rubia cordifolia radix, Scutellariae barbatae herba, and Fritillariae thunbergii bulbi. The external washing 
was done with Astragali membranaceus radix, Angelica sinensis radix, Paeoniae rubra radix, Lumbricus terrestris, Ligustici chuanxiong rhizoma, Prunus persicae semen, and Carthami flos.

The incidence of CIPN was almost half as high in the patients treated additionally with Chinese herbs as evaluated by clinical testing $(P<0.05)[94]$.

3.2.4. Geranii herba plus Aconiti radix. External application of a combination of Geranii herba and Aconiti radix extract has been shown to be effective in a rat model of oxaliplatin evoked neuropathy. Mechanical allodynia and thermal hyperalgesia were alleviated. NGF was increased, substance $\mathrm{P}$ decreased in the group treated with Geranii herba and Aconiti radix extract additionally to oxaliplatin compared to oxaliplatin alone [95].

In the following randomized clinical study 58 patients with CIPN from oxaliplatin, taxol, or capecitabine were assigned prospectively in a controlled randomized trial: 30 patients were assigned to the study group and 28 were used as a control. The clinical study revealed that symptoms of pain, paraesthesia, and swelling were relieved after one week of therapy and it was concluded that Geranii herba plus Aconiti radix granule can relieve neuropathy and improve the quality of life. Unfortunately the authors did not provide data in the published abstract, which species of Geranii herba or Aconiti radix they used [95].

3.2.5. Goshajinkigan (Jap.) $=$ Niu Che Sen Qi Wan (Chin.) $=$ Pilula renales plantaginis et achyranthis (Lat.) $=$ Life Preserving Kidney Qi Pill (Engl.). This formula derives from the Jisheng Fang, written by Yan Yonghe, a little known but highly regarded physician of the Song Dynasty, published in 1253 [125]. In Japanese Kampo medicine it is called Goshajinkigan (GJG) and is frequently used as a standardized granule. It contains 10 different herbs (Rehmannia viride radix, Achyranthis bidentatae radix, Corni fructus, Dioscorea opposita rhizoma, Plantaginis semen, Alismatis rhizoma, Moutan cortex, Cinnamomi cortex, Aconiti lateralis praeparata tuber, and Poria alba) [126]. GJG has antioxidant properties [127, 128].

GJG was tested for its effect on CIPN in animal studies. In a rat model of oxaliplatin-induced neuropathy repeated administration of GJG prevented the oxaliplatin-induced cold hyperalgesia but not mechanical allodynia and axonal degeneration of the rat sciatic nerve. A single administration of GJG reduced both cold hyperalgesia and mechanical allodynia after the development of neuropathy. GJG did not affect the antitumour effect of oxaliplatin on the tumour cells or mice implanted with tumour cells [98].

GJG was also tested in a rat model for paclitaxel-induced peripheral neuropathy, but as with oxaliplatin no regeneration was found in histological examination [96]. Nevertheless further rat animal studies showed a positive effect of GJG on cold allodynia [97].

GJG has been widely used to treat symptoms like numbness, vibration sensation, cold sensation, and limb pain associated with diabetic neuropathy [129].
It has been shown to prevent oxaliplatin-induced peripheral neuropathy in a FOLFOX-regimen (FOL: Folinic acid (leucovorin), F: Fluorouracil (5-FU), OX: Oxaliplatin (Eloxatin)) in clinical studies $[99,100]$. In a noncontrolled study 14 patients received GJG every day after the first oxaliplatin infusion. GJG seemed to prevent acute oxaliplatin-induced neurotoxicity [99]. In a retrospective analysis of 45 patients, 22 received GJG during their FOLFOX regimen against nonresectable or recurrent colorectal cancer, while $23 \mathrm{did}$ not get this additional therapy. The incidence of grade 3 $\mathrm{PN}$ in the GJG group was significantly lower than in the control group $(P<0.01$, log-rank test $)$. The incidence of grade $3 \mathrm{PN}$ after 10 courses of chemotherapy was $0 \%$ in the GJG group and $12 \%$ in the control group, and after 20 courses was $33 \%$ in the GJG group and $75 \%$ in the control group [100]. A further retrospective analysis was performed in 90 patients who were given a FOLFOX regimen for metastatic colorectal cancer. Two treatment groups were compared: FOLFXOX plus GJG and FOLFOX plus GJG plus $\mathrm{Ca}^{2+} / \mathrm{Mg}^{+}$, and two control groups: FOLFOX without additional therapy and FOLFOX plus $\mathrm{Ca}^{2+} / \mathrm{Mg}^{+}$. When a cumulative dose of oxaliplatin exceeded $500 \mathrm{mg} / \mathrm{m}^{2}$, the incidence of $\mathrm{PN}$ was $91 \%$ in the FOLFOX without additional therapy group, $100 \%$ in the FOLFOX group with additional $\mathrm{Ca}^{2+} / \mathrm{Mg}^{+}$therapy, and $79 \%$ in the FOLFOX plus GJG plus $\mathrm{Ca}^{2+} / \mathrm{Mg}^{+}$therapy group and $50 \%$ in the GJG plus FOLFOX group. The cumulative oxaliplatin dose when $50 \%$ of patients developed neurotoxicity was $765 \mathrm{mg} / \mathrm{m}^{2}$ in the GJG plus FOLFOX and $340 \mathrm{mg} / \mathrm{m}^{2}$ in the FOLFOX plus GJG plus $\mathrm{Ca}^{2+} / \mathrm{Mg}^{+}$group, respectively, and $255 \mathrm{mg} / \mathrm{m}^{2}$ in both control groups. The authors concluded that concomitant administration of GJG reduced the neurotoxicity of oxaliplatin without having a negative influence on the response rate [101], so for further validation of these data a concept for a prospective, controlled, double blinded randomized study was developed [130].

GJG was also tested for paclitaxel-induced neuropathy in breast and gynecological cancer. A retrospective study on 82 patients found that GJG was possibly effective for the treatment and the prevention of peripheral neuropathy and seemed to be more effective when administered from the beginning of chemotherapy using paclitaxel [102].

In a prospective study in paclitaxel/carboplatin treated patients with ovarian or endometrial cancer, patients were randomly divided into two groups. 14 patients received vitamin $\mathrm{B} 12$ and 15 patients vitamin B12 plus GJG. The observation period was 6 weeks following treatment initiation. A NCI-CTCAE (National Cancer Institute-Common Toxicity criteria) grade 3 of neurotoxicity developed in 2 patients $(14.3 \%)$ after 6 weeks in the vitamin B12, whereas no neurotoxicity was observed in the vitamin B12/GJG group. The change in the frequency of abnormal current perception threshold (CPT) ratio was significantly lower in the VB12/GJG group in comparison to VitB12 alone $(P<$ 0.05 ), which suggests that GJG inhibits the progression of PN [103]. 
3.2.6. Keishikajutsubuto (Jap.) = Gui Zhi Jia Shu Fu Tang (Chin.) = Decoctum ramulorum cassiae cum atractylodis macrocephae et aconiti $($ Lat. $)=$ Cinnamon Twig Decoction plus Atractylodes and Aconite (Engl.). This formula has its basis from the Shang Han Lun and was further developed as a Japanese experimental formula during the Edo period (1603 to 1868). It contains Cinamomi cortex, Aconiti lateralis praeparata tuber, Zingiberis rhizoma, Jujubae fructus, Glycyrrhizae radix, and Atracylodis macrocephalae rhizoma.

This herbal combination with an increased dose of Aconiti lateralis praeparata tuber was used as a granule for 11 patients with metastatic colorectal cancer receiving FOLFOX in a noncontrolled study. Reduction of neuropathy was observed in 5 cases after chemotherapy (45.5\%) [104].

The same herbal granule was also used in a study on postherpetic neuralgia and was found to be effective. In three of 15 patients in this noncontrolled trial continuation of the treatment with Keishikajutsubuto was not possible due to hot flashes or gastric discomfort. The remaining 12 patients showed a VAS improvement rate of $76.5 \pm 27.7 \%$ (mean \pm standard deviation) [131].

3.2.7. Ogikeishigomotsuto (Jap.) = Huang Qi Wu Wu Tang $($ Chin. $)=($ Decotum quinque medicamentorum cum astragalo $($ Lat. $)=$ Astragalus and Cinnamon Five Herb Combination (Engl.). This classical combination derives from Jin Gui Yao Lue (Essential Prescriptions from the Golden Chamber) [132]. In Kampo medicine it is called Ogikeishigomotsuto, containing Astragali membranaceus radix, Cinnamomi cortex, Paeonia alba radix, Jujubae fructus, and Zingiberis rhizoma. It has been used in individual cases for neuropathic pain due to ANCA-associated vasculitis [133].

In single case report the granule showed a positive effect on neuropathic pain and it allowed the continuation of the suspended chemotherapy with oxaliplatin [105].

3.2.8. Shakuyakukanzoto (Jap.) = Shao Yao Gan Cao Tang (Chin.), Formula glycyrrhizae et paeonia (Lat.), Peony and Licorice Decoction (Engl.). This classical formula derives from the Shang Han Lun [124]. In Kampo medicine it is called Shakuyakukanzoto and it is a herbal granule of Paeonia alba radix and Glycyrrhizae radix. It is used to relieve menstrual pain and muscle spasm as well as muscle pain due to the chemotherapeutic agents paclitaxel and carboplatin [134136] and has also been tested for CIPN. A retrospective case analysis of 23 patients showed a positive effect on neuropathic pain in CIPN after paclitaxel for ovarian carcinoma [107].

This was supported by animal studies in a mouse model of paclitaxel-induced pain. Shakuyakukanzoto significantly relieved the allodynia and hyperalgesia induced by paclitaxel [106]. Shakuyakukanzoto has also been tested for preventing neurotoxic side effects of FOLFOX and the effect was retrospectively compared to the treatment with GJG (see Section 3.2.5). 44 patients with metastatic colorectal cancer received FOLFOX and concurrently received either GJG ( $n=$ $20)$ or Shakuyakukanzoto $(n=24)$. The response was $50.0 \%$ in the GJG and 65\% in the Shakuyakkanzoto group. The authors concluded that both recipes are able to reduce the FOLFOXinduced neurotoxicity [108].

3.3. Herbs Tested or Herbal Ingredients for Neuropathic Pain, Not Specifically Tested to CIPN. Capsaicin is the active component of chili peppers, which are plants belonging to the genus Capsicum. Topical creams with capsaicin are used to treat peripheral neuropathic pain. Following application to the skin capsaicin causes enhanced sensitivity, followed by a period with reduced sensitivity and, after repeated applications, persistent desensitisation.

Topical capsaicin is used to treat postherpetic neuralgia and HIV-neuropathy and has been found to be effective in multiple trials. There are risks of epidermal innervation upon repeated application over long periods [137].

Aconiti lateralis praeparata radix is a herb used in many recipes for neuropathy like GJG (see Section 3.2.5) and Keishikajutsubuto (see Section 3.2.6) and is often used for several types of persistent pain. In a mouse model analgesic effects caused by inhibition of astrocytic activation by Aconiti lateralis praeparata radix were mimicked by the intrathecal injection of fluorocitrate. The study indicated that the activation of spinal astrocytes was responsible for the late maintenance phase of neuropathic pain, so Aconiti lateralis praeparata radix could be a therapeutic strategy for treating neuropathic pain [138]. In a rat model Aconiti lateralis praeparata radix was tested against a placebo for allodynia and thermal hyperalgesia. A dose-dependent effect was measured. The effects were inhibited by intraperitoneal and intrathecal nor-binaltorphimine, a selective kappa-opioid receptor antagonist, but not by intraperitoneal naloxone. The authors concluded that the effect against neuropathic pain is induced via spinal kappa-opioid receptor mechanisms [139].

Moutan cortex and Coicis semen have been tested positively in two different neuropathic pain mice models. In one model allodynia was induced by intrathecal administration of prostaglandin F2alpha (PGF2alpha) and in the second model by selective $\mathrm{L} 5$ spinal nerve transection. The extracts of Moutan cortex and Coicis semen dose dependently alleviated the PGF2alpha-induced allodynia. The increase in NADPH diaphorase activity in the spinal cord associated with neuropathic pain was also blocked by these extracts. These results suggest that Moutan cortex and Coicis semen are substances effective in the treatment of neuropathic pain [140].

Nigella sativa and one main compound thymoquinone were beneficial on histopathological changes of sciatic nerves in streptozotocin (STZ) induced diabetic rats. Evaluation of the tissues in the diabetic animals showed fewer morphologic alterations, and myelin breakdown decreased significantly after treatment with Nigella sativa and thymoquinone. The ultrastructural features of axons also showed improvement [141].

Ocimum sanctum was investigated in sciatic nerve transection induced peripheral neuropathy in rats. Axonal degeneration was assessed histopathologically. Paw pressure, Von Frey Hair, tail cold-hyperalgesia, and motor in-coordination tests were performed to assess the in vivo extent of neuropathy. Biochemical estimations of thiobarbituric acid reactive 
species (TBARS), reduced glutathione (GSH), and total calcium levels were also performed. Ocimum sanctum attenuated axonal degeneration, rise in TBARS, total calcium, and decrease in GSH levels in a dose-dependent manner. In vivo reduction of nociceptive threshold and motor incoordination was found. The authors concluded that antioxidant and calcium attenuating actions may be responsible for the amelioration [142].

In an another animal study STZ-induced diabetic rats received intraperitoneal injection of this extract of Teucrium polium. The treated rats exhibited a lower nociceptive score as compared to untreated diabetic rats. The results may suggest a therapeutic potential of Teucrium polium for the treatment of hyperalgesia [143].

A hexanic extract from Phyllanthus amarus has been shown to be effective in a neuropathic model of nociception. The antiallodynic effects seemed not to be associated with the impairment of motor coordination or with the development of tolerance. Apart from its anti-inflammatory actions, which are probably linked to the presence of lignans, another as yet unidentified active principle(s) present in the hexanic extract of Phyllanthus amarus produces pronounced anti-allodynia [144].

An aqueous extract of Sesbania sesban was tested in STZinduced diabetic rats. The treated group showed an increased tail flick latency significantly when compared with pregabalin and reduced superoxide anion and total calcium levels which gave evidence of neuroprotective effects [145].

\section{Discussion}

In spite of intense research in the last decades, no conventional pharmacological substance has been established as a sufficient and safe treatment of CIPN-induced neuroprotection and regeneration [31, 43-63, 65-67]. Herbal treatment is commonly used for different kind of therapies where western medicine does not offer a sufficient efficacy, but the evidence of the use of herbal treatment is not clear and has to be elucidated.

One main problem of doing research on herbs and understanding the mechanisms of their action is the fact that herbs contain a number of active compounds and by tradition, especially in Asian herbal therapy, combinations of multiple herbs are common.

Classical research mainly focusing on a single active compound has been done often without regard to historical knowledge of the therapeutic utility of the plant source [146]. This does not reflect the complexity of traditional Asian herbal recipes, while there is some evidence that single components extracted from plants are less potent than the complete extract [147] and a multitarget approach might be more effective than a single target approach [148]. Pharmacological mixtures can also have the advantage of potentiating the action of their multiple bioactive components and the option of an individualized therapy $[149,150]$. For scientific understanding of the mechanisms of action there is still a need for basic research on single herbs and their active compounds, but research should not stop at this level but continue with research of multicompounds, their interactions, and increasing or decreasing activity in combinations.

The positive effect of a single herb on neuroprotection or regeneration was not found in any clinical study and rarely found in animal studies. Only the flavonoid apigenin from Salvia officinalis seems to protect neurons against toxicity [116] and Ginkgo biloba as a single herb shows some evidence of preventing neuronal degeneration and inducing neural regeneration in CIPN [109-111].

Mainly in animal studies only a few single herbs have been tested for treatment of CIPN improving neuropathic pain. This effect might be induced by Acorus calamus rhizoma due to its antioxidative, anti-inflammatory, and calcium inhibitory actions [70]. Flavonoids from Matricaria chamomilla have an antispasmodic and an anti-inflammatory effect $[83,84]$. Salvia officinalis is probably working due to its antioxidant activity and anti-inflammatory properties [112-115] and is also involved in an opioid mechanism [119]. Fritillaria bulbus might be effective due to its isosteroidal alkaloid verticinone [91]. While Cannabis sativa is effective in multiple and central pain syndromes [72-78], the fact that two Cannabis agonists had been shown to be effective in CIPN in a rat model could be a hint that Cannabis sativa might be a promising substance for pain in CIPN in the future.

Only Ginkgo biloba extract has been positively tested in a small retrospective clinical study in humans [87] and Sweet bee venom had shown positive results by injection into acupuncture points in a small clinical case series $[89,90]$. Topical capsaicin from chili peppers was found to be effective in multiple trials to treat pain from postherpetic neuralgia and HIV-neuropathy but has not been specifically tested against pain in CIPN [137].

There are a few more single herbs tested in animal models for the treatment of neuropathic pain in other conditions than CIPN. Moutan cortex and Coicis semen have been tested positively in two different neuropathic pain models. Both herbs dose dependently alleviated the PGF2alpha-induced allodynia and blocked NADPH diaphorase activity in the spinal cord associated with neuropathic pain [140].

Nigella sativa, Ocimum sanctum, Teucrium polium, Phyllanthus amarus, and Sesbania sesban also have been tested in single studies to ameliorate neuropathic pain [141-145].

Aconiti lateralis praeparata radix in a mouse model of CIPN had analgesic effects by inhibition of spinal astrocytic activation [138] and in a rat model Aconiti lateralis praeparata radix had a dose-dependent effect on allodynia and thermal hyperalgesia which was induced via spinal kappa-opioid receptor mechanisms [139], which confirmed similar animal studies on neuropathic pain in diabetic mice [148]. Active components are alkaloids, for example, aconitine and mesaconitine and have in addition pain-relieving effects, cardiotonic and vasodilator actions [151-155].

Aconiti lateralis praeparata radix is often used for several types of persistent pain while it is rarely used as a single herb for CIPN. But interestingly Aconiti lateralis praeparata radix is part of many recipes used for CIPN as GJG, Shakuyakukanzoto, Keishikajutsubuto, and Geranii herba plus Aconiti radix combination (see Sections 3.2.4, 3.2.5, 3.2.6, and 3.2.8). 
Some of the pain-relieving effects of GJG are known to be induced by aconite [139] and it is considered that the analgesic effect is exerted by the suppression of pain-transmitting substances release by $\kappa$-opioid receptor stimulation mediated by dynorphin, an endogenous opioid substance released by processed aconite [148]. But on the other hand, these effects were stronger, when using of the full recipe in comparison to the use of Aconiti lateralis praeparata radix alone [155].

GJG is the best tested herbal recipe for CIPN. But not all details of its mechanism have been clearly identified and for some ingredients the effects are unknown. It is considered that the analgesic effect is exerted through the improvement of peripheral nocireceptor sensitivity, vasodilation, and peripheral circulation by the promotion of $\mathrm{NO}$ production due to the effects of Alismatis rhizoma and Dioscorea opposita rhizoma mediated by the bradykinin B2 receptor and the muscarinic acetylcholine receptors [148]. Another substance from GJG, Rehmanniae praeparata radix, could promote the function of learning and memory of MSG rats, and its mechanism may be related to the increase of the expression of hippocampal c-fos and nerve growth factor (NGF) [156], which can be relevant for regeneration in CIPN as well, while NGF exhibits potent biological activities such as preventing neuronal death, promoting neurite outgrowth, and supporting synapse formation [157]. One further mechanism of GJG might be its positive effect on improving the microcirculation, which might be helpful for the recovery of the nerves in CIPN [158].

Even though the full recipe was clinically positively tested in a couple of clinical trials, most of them were uncontrolled or retrospective analyses $[99-102,130]$ and the only prospective trial had a limited number of participants [103]. So the evidence of a positive effect is still low and needs further clinical and experimental confirmation.

GJG has also been compared to another herbal recipe, Shakuyakukanzoto, and both were found to be effective in the treatment of CIPN, but there was no negative control group [107]. Shakuyakukanzoto was analyzed in a mouse model [106] and in a retrospective analysis of 23 cases without controls [108]. Since Shakuyakukanzoto contains only two herbs, Paeonia alba radix and Glycyrrhizae radix, the future evaluation of its role for treating CIPN might be easier than for other herbal recipes. While neuronal apoptosis can be triggered by oxidative stress and mitochondrial dysfunction, substances with an antioxidative potency are possible candidates for treatment of CIPN [19-22], so the fact that paeoniflorin as one bioactive component of Paeonia alba radix has been positively tested as an antioxidant in a nonneuronal cell model might be relevant [159]. It has also neuroprotective effects which are closely correlated to activating the adenosine A1 receptor, ameliorating the function of the cholinergic nerve, regulating ion channel homeostasis, retarding oxidative stress, apoptosis of the neurocytes, and promoting nerve growth [160]. Paeonia alba radix was used in another recipe (see Section 3.2.7) and in two recipes Paeonia rubra radix (see Sections 3.2.1 and 3.2.2) has been used. Paeonia alba radix and Paeonia rubra radix have a lot of similarities in their components and paeoniflorin is a bioactive compound of both herbs [161].
While the reason for using herbal recipes derives from historical knowledge or transferral of historical concepts to modern diseases, interestingly four of eight herbal recipes tested for CIPN contain Glycyrrhizae radix. To find the scientific ratio behind this decision, analyses of its action in the context of neural cell damage might be fruitful.

Another herb that has been used in four of eight herbal recipes tested for CIPN is Astragalus membranaceus radix. This might be rational, while Astragalus membranaceus radix water extracts caused a marked enhancement of the NGF-mediated neurite outgrowth and the expression of growth-associated protein 43 from PC12 cells in vitro [162]. Astragalus membranaceus radix extracts can be a potential nerve growth-promoting factor, being salutary in aiding the growth of axons in the peripheral nerve [162]. Astragaloside IV (AGS-IV), one bioactive compound of Astragalus membranaceus radix, is an aldose-reductase inhibitor and a freeradical scavenger which suppressed a decrease in myelinated fibers, promoted an increase in myelinated fiber density and an increase in segmental demyelination in diabetic rats [163]. It also increased the activity of glutathione peroxidase in nerves, depressed the activation of aldose reductase in erythrocytes, decreased the accumulation of advanced glycation end products in both nerves and erythrocytes, and elevated $\mathrm{Na}+, \mathrm{K}+$-ATPase activity in both the nerves and erythrocytes of diabetic rats, so it is considered to be protective against the progression of peripheral neuropathy [163].

The herbal recipe Bu Yang Huan and a modified extension have been used in two clinical controlled randomized trials [92, 93] (3.2.1 and 3.2.2, see above) and was found to be effective in both. The formula used is very complex, and little is known about the mechanism of its compound, but herbs like Astragalus membranaceus radix, Paeonia rubra radix, and Dioscorea opposita rhizoma are part of the herbal combination where possible mechanisms are described (3.2.1, 3.2.2, 3.2.5, 3.2.7, see above), so at least something is known about its possible mechanisms of effect.

Another study used a modified classical prescription named Chai Hu Long Gu Mu Li Wan (see Section 3.2.3) and found positive results as well [94]. Even though it is a controlled randomized trial, the treatment protocol is very complicated, combining oral intake of medical herbs and external washing with other herbs. So also with this herbal combination little is known about the mechanism of its action.

Two other herbal recipes have been reported for successful clinical use, Keishikajutsubuto = Gui Zhi Jia Shu Fu Tang (see Section 3.2.6) and Ogikeishigomotsuto = Huang Qi $W u$ Wu Tang (see Section 3.2.7). But these studies are either uncontrolled or a case report, so the quality of the evidence is very low.

The trials on the single herbs and herbal combinations tested so far do not provide a clear recommendation for clinical use. But research on some single herbs as well as on combinations like GJG is promising, even though the level of knowledge is limited to basic research on the mechanisms of action and evidence from clinical trials. But it is necessary to continue research on this field, while treatment concepts for CIPN are lacking. 
The most used herbs of herbal recipes in clinical trials on CIPN are Aconiti lateralis praeparata radix, Rehmannia praeparata radix, Paeonia (alba and rubra) radix, Astragalus membranaceus radix, and Glycyrrhizae radix.

In Chinese medicine experience Astragalus membranaceus radix is supporting the $\mathrm{Q} i$, which means in western terms supporting the general energy level of the body, which is usually reduced in CIPN by activating the vegetative nervous system. Rehmannia praeparata radix is basically supporting the Yin, which in western terms reflects the structural damage of tissue, in the case of CIPN the peripheral nerves. Paeonia alba radix and Paeonia rubra radix promote the flow of the Xue, which in western terminology has its correlate in enhancing the microcirculation which might be reduced in CIPN; promotion of the perfusion might enhance the regeneration.

Aconiti lateralis praeparata radix supports in Chinese medicine theory the Yang. A typical symptom of Yang deficiency is ice-coldness, which is prominent in the extremities in some cases of CIPN. Glycyrrhizae radix supports the fluids and has a balancing effect on the whole recipe.

So in spite of using different terms, Chinese medicine theory describes physical reactions of the body that can be explained by western physiology and has found its proof by experimental studies [138, 139, 148, 151-156, 159-164].

So using these herbs has as rational foundation on the basis of Chinese medical theory as well as from experimental studies, but unfortunately still little is known about the complex physiological mechanisms of herbal combinations, the interactions of the substances, and the mechanisms of action of many other substances used in herbal medicine. The challenge for future research is bringing historical knowledge and modern scientific analysis together.

Due to this limited knowledge on the mechanisms of the action of herbs, we additionally collected data on herbs that are a putative treatment option for CIPN. While oxidative stress and mitochondrial dysfunction promote CIPN, substances with an antioxidative potency are possible candidates for the treatment of CIPN. So in the list below we list herbal antioxidants tested in neuronal cell or disease models. Additionally we added herbs for enhancement of nerve growth as putative treatment options for CIPN. NGF exhibits potent biological activities such as preventing neuronal death, promoting neurite outgrowth, and supporting synapse formation [157], which has a relevance for the development of CIPN [39].

Putative Herbs or Herbal Compounds for the Treatment of CIPN

(1) Herbal Antioxidants Tested in Neuronal Cell or Disease Models. Puerarin (from Pueraria lobata radix) [165, 166], Icariin (from Epimedii herba) [167], a fraction of polysaccharides (from Lycium barbarum) [168], Ginsenoside Rg1 (from Notoginseng panacis) [169], honokiol and magnolol (from Magnolia officinalis) [170], Ginkgolide A, B (from Ginkgo biloba) [171], huperzine A (from Huperzia serrata)
[171], Ginseng radix [172], Notoginseng panacis radix [173], 3,5,4' -tetrahydroxystilbene-2-O-beta-Dglucoside (from Polygonum multiflorum) [174-176], celastrol (from Tripterygium wilfordii Hook) [177], Salvianolic acid B (from Salvia miltiorrhiza) [178, 179], tanshinone IIA (from Salvia miltiorrhizae) [180], Gastrodia elata rhizoma [181, 182], Astragaloside IV (from Astragalus membranaceus radix) [163], Tetramethylpyrazine (from Ligusticum wallichi) [183, 184], Ziziphus spinosus semen [185], Baicalein (from Scutellaria baicalensis radix Georgi) [186], Uncaria rhynchophylla [164], 6,7-dihydroxy2-methoxy-1,4-phenanthrenedione, chrysoeriol $4^{\prime}$ O-beta-D-glucopyranoside, chrysoeriol 7-O-beta-Dglucopyranoside, and alternanthin (from Dioscorea opposita radix) [187].

(2) Herbs or Herbal Compounds That Enhance Nerve Growth. Cistanches herba $[157,188]$, Huperzine A (HupA) from Huperzia serrata [189, 190], a lipophilic fraction of panax ginseng [191]. Astragalus membranaceus radix [162], Gentiside C, a compound of Gentiana rigescens radix [191], Rehmanniae praeparata radix [192], Paeoniflorin of Paeonia alba radix [193].

Research with the aim of proving the benefits of promising substances or herbal combinations has to describe the mechanism of action for single herbs and single components, investigate the interactions of combinations of substances, and analyse the promoting effects of combinations.

From this viewpoint, research in this field has not very much progressed, but if research does not provide these data, herbal combinations will not find acceptance in mainstream treatments in non-Asian countries in Europe, North America, or Australia.

This challenge could be taken if more international cooperation of interested research groups would be organized.

\section{Conclusion}

Experimental and clinical studies have not yielded enough evidence to establish a standard practice for the treatment of CIPN, but from this literature review, a lot of promising substances, mainly Chinese medical herbs with possible effect in CIPN or a putative influence on mechanisms of CIPN, have been identified in the last years.

The knowledge of the mechanisms of action is still limited and the quality of the clinical trials needs further improvement. In the future not only the mechanisms of action for single herbs and single components have to be described, but interactions of combinations of substances as well as interactions with chemotherapy have to be investigated and analysed in depth. While CIPN has multiple possible mechanisms of neuronal degeneration, a combination of components might be a promising opportunity focusing on multiple targets of degeneration or activating regeneration. 


\section{References}

[1] G. Wilkes, "Peripheral neuropathy related to chemotherapy," Seminars in Oncology Nursing, vol. 23, no. 3, pp. 162-173, 2007.

[2] R. B. Lipton, S. C. Apfel, J. P. Dutcher et al., "Taxol produces a predominantly sensory neuropathy," Neurology, vol. 39, no. 3, pp. 368-373, 1989.

[3] J. M. A. van Gerven, J. W. B. Moll, M. J. van den Bent et al., "Paclitaxel (Taxol) induces cumulative mild neurotoxicity," European Journal of Cancer A, vol. 30, no. 8, pp. 1074-1077, 1994.

[4] C. Visovsky, M. Collins, L. Abbott, J. Aschenbrenner, and C. Hart, "Putting evidence into practice: evidence-based interventions for chemotherapy-induced peripheral neuropathy," Clinical Journal of Oncology Nursing, vol. 11, no. 6, pp. 901-913, 2007.

[5] J. W. Hay, "Quality of life effects of chemotherapy-induced neuropathy in ovarian cancer," Proceedings of the American Society of Clinical Oncology, vol. 21, abstract 886, p. 222a, 2002.

[6] R. B. Lipton, S. C. Apfel, J. P. Dutcher et al., "Taxol produces a predominantly sensory neuropathy," Neurology, vol. 39, no. 3, pp. 368-373, 1989.

[7] F. H. Hausheer, R. L. Schilsky, S. Bain, E. J. Berghorn, and F. Lieberman, "Diagnosis, management, and evaluation of chemotherapy-induced peripheral neuropathy," Seminars in Oncology, vol. 33, no. 1, pp. 15-49, 2006.

[8] A. M. Corse and R. W. Kunel, "Peripheral neuropathy," in Principles of Ambulatory Medicine, L. R. Barker, J. R. Burton, and P. D. Zieve, Eds., pp. 1296-1314, Williams \& Wilkins, Baltimore, Md, USA, 5th edition, 1999.

[9] Oxaliplatin Prescribing Information. Drugs.com. Hospira Worldwide, 2013, http://www.drugs.com/pro/oxaliplatin.html.

[10] F. H. Hausheer, R. L. Schilsky, S. Bain, E. J. Berghorn, and F. Lieberman, "Diagnosis, management, and evaluation of chemotherapy-induced peripheral neuropathy," Seminars in Oncology, vol. 33, no. 1, pp. 15-49, 2006.

[11] V. Chaudhry, M. Chaudhry, T. O. Crawford, E. SimmonsO'Brien, and J. W. Griffin, "Toxic neuropathy in patients with pre-existing neuropathy," Neurology, vol. 60, no. 2, pp. 337-340, 2003.

[12] S. Mielke, K. Mross, T. A. Gerds et al., "Comparative neurotoxicity of weekly non-break paditaxel infusions over 1 versus $3 \mathrm{~h}$," Anti-Cancer Drugs, vol. 14, no. 10, pp. 785-792, 2003.

[13] A. de Gramont, C. Boni, M. Navaro et al., "Oxaliplatin/5FU/LV in the adjuvant treatment of stage II and stage III colon cancer: efficacy results with a medican follow-up of 4 years," Journal of Clinical Oncology, vol. 23, p. 16, 2005.

[14] A. Grothey, "Clinical management of oxaliplatin-associated neurotoxicity," Clinical Colorectal Cancer, vol. 5, no. 1, pp. S38S46, 2005.

[15] R. W. Kuncl and E. B. George, "Toxic neuropathies and myopathies," Current Opinion in Neurology, vol. 6, no. 5, pp. 695-704, 1993.

[16] S. Quasthoff and H. P. Hartung, "Chemotherapy-induced peripheral neuropathy," Journal of Neurology, vol. 249, no. 1, pp. 9-17, 2002.

[17] R. W. Gregg, J. M. Molepo, V. J. A. Monpetit et al., "Cisplatin neurotoxicity: the relationship between dosage, time, and platinum concentration in neurologic tissues, and morphologic evidence of toxicity," Journal of Clinical Oncology, vol. 10, no. 5, pp. 795-803, 1992.
[18] J. S. Gill and A. J. Windebank, "Cisplatin-induced apoptosis in rat dorsal root ganglion neurons is associated with attempted entry into the cell cycle," The Journal of Clinical Investigation, vol. 101, no. 12, pp. 2842-2850, 1998.

[19] M. S. Yoon, Z. Katsarava, M. Obermann et al., "Erythropoietin overrides the triggering effect of DNA platination products in a mouse model of Cisplatin-induced neuropathy," BMC Neuroscience, vol. 10, article 77, 2009.

[20] E. K. Joseph and J. D. Levine, "Mitochondrial electron transport in models of neuropathic and inflammatory pain," Pain, vol. 121, no. 1-2, pp. 105-114, 2006.

[21] E. K. Joseph and J. D. Levine, "Comparison of oxaliplatin- and Cisplatin-induced painful peripheral neuropathy in the rat," The Journal of Pain, vol. 10, no. 5, pp. 534-541, 2009.

[22] E. K. Joseph, X. Chen, O. Bogen, and J. D. Levine, "Oxaliplatin acts on IB4-positive nociceptors to induce an oxidative stressdependent acute painful peripheral neuropathy," The Journal of Pain, vol. 9, no. 5, pp. 463-472, 2008.

[23] G. Cavaletti, G. Tredici, M. Braga, and S. Tazzari, "Experimental peripheral neuropathy induced in adult rats by repeated intraperitoneal administration of taxol," Experimental Neurology, vol. 133, no. 1, pp. 64-72, 1995.

[24] C. M. Peters, J. M. Jimenez-Andrade, M. A. Kuskowski, J. R. Ghilardi, and P. W. Mantyh, "An evolving cellular pathology occurs in dorsal root ganglia, peripheral nerve and spinal cord following intravenous administration of paclitaxel in the rat," Brain Research, vol. 1168, no. 1, pp. 46-59, 2007.

[25] O. A. Shemesh and M. E. Spira, "Paclitaxel induces axonal microtubules polar reconfiguration and impaired organelle transport: implications for the pathogenesis of paclitaxelinduced polyneuropathy," Acta Neuropathologica, vol. 119, no. 2, pp. 235-248, 2010.

[26] L. H. Weimer, "Medication-induced peripheral neuropathy," Current Neurology and Neuroscience Reports, vol. 3, no. 1, pp. 86-92, 2003.

[27] K. D. Tanner, J. D. Levine, and K. S. Topp, "Microtubule disorientation and axonal swelling in unmyelinated sensory axons during vincristine-induced painful neuropathy in rat," Journal of Comparative Neurology, vol. 395, pp. 481-492, 1998.

[28] K. S. Topp, K. D. Tanner, and J. D. Levine, "Damage to the cytoskeleton of large diameter sensory neurons and myelinated axons in vincristine-induced painful peripheral neuropathy in the rat," Journal of Comparative Neurology, vol. 424, no. 4, pp. 563-576, 2000.

[29] Z. Sahenk, S. T. Brady, and J. R. Mendell, "Studies on the pathogenesis of vincristine-induced neuropathy," Muscle and Nerve, vol. 10, no. 1, pp. 80-84, 1987.

[30] S. Lobert, B. Vulevic, and J.-J. Correia, "Interaction of vinca alkaloids with tubulin: a comparison of vinblastine, vincristine, and vinorelbine," Biochemistry, vol. 35, no. 21, pp. 6806-6814, 1996.

[31] A. A. Argyriou, P. Marmiroli, G. Cavaletti, and H. P. Kalofonos, "Epothilone-induced peripheral neuropathy: a review of current knowledge," Journal of Pain and Symptom Management, vol. 42, no. 6, pp. 931-940, 2011.

[32] R. J. Kowalski, P. Giannakakou, and E. Hamel, "Activities of the microtubule-stabilizing agents epothilones A and B with purified tubulin and in cells resistant to paclitaxel (Taxol)," The Journal of Biological Chemistry, vol. 272, no. 4, pp. 2534-2541, 1997. 
[33] K. H. Altmann, M. Wartmann, and T. O'Reilly, "Epothilones and related structures-a new class of microtubule inhibitors with potent in vivo antitumor activity," Biochimica et Biophysica Acta, vol. 1470, no. 3, pp. M79-M91, 2000.

[34] A. A. Argyriou, G. Iconomou, and H. P. Kalofonos, "Bortezomib-induced peripheral neuropathy in multiple myeloma: a comprehensive review of the literature," Blood, vol. 112, no. 5, pp. 1593-1599, 2008.

[35] G. Cavaletti and E. Nobile-Orazio, "Bortezomib-induced peripheral neurotoxicity: still far from a painless gain," Haematologica, vol. 92, no. 10, pp. 1308-1310, 2007.

[36] G. Cavaletti, A. Gilardini, A. Canta et al., "Bortezomib-induced peripheral neurotoxicity: a neurophysiological and pathological study in the rat," Experimental Neurology, vol. 204, no. 1, pp. 317$325,2007$.

[37] I. Casafont, M. T. Berciano, and M. Lafarga, "Bortezomib induces the formation of nuclear poly(A) RNA granules enriched in Sam68 and PABPN1 in sensory ganglia neurons," Neurotoxicity Research, vol. 17, no. 2, pp. 167-178, 2010.

[38] T. H. Landowski, C. J. Megli, K. D. Nullmeyer, R. M. Lynch, and R. T. Dorr, "Mitochondrial-mediated disregulation of $\mathrm{Ca}^{2+}$ is a critical determinant of Velcade (PS-341/Bortezomib) cytotoxicity in myeloma cell lines," Cancer Research, vol. 65, no. 9, pp. 3828-3836, 2005.

[39] C. Montagut, A. Rovira, and J. Albanell, "The proteasome: a novel target for anticancer therapy," Clinical and Translational Oncology, vol. 8, no. 5, pp. 313-317, 2006.

[40] C. H. Chung, J. Aulino, N. J. Muldowney et al., "Nuclear factorkappa B pathway and response in a phase II trial of bortezomib and docetaxel in patients with recurrent and/or metastatic head and neck squamous cell carcinoma," Annals of Oncology, vol. 21, no. 4, pp. 864-870, 2010.

[41] J. P. Cata, H. R. Weng, A. W. Burton, H. Villareal, S. Giralt, and P. M. Dougherty, "Quantitative sensory findings in patients with bortezomib-induced pain," The Journal of Pain, vol. 8, no. 4, pp. 296-306, 2007.

[42] E. R. Lepper, N. F. Smith, M. C. Cox, C. D. Scripture, and W. D. Figg, "Thalidomide metabolism and hydrolysis: mechanisms and implications," Current Drug Metabolism, vol. 7, no. 6, pp. 677-685, 2006.

[43] G. Cavaletti and P. Marmiroli, "Chemotherapy-induced peripheral neurotoxicity," Nature Reviews Neurology, vol. 6, no. 12, pp. 657-666, 2010.

[44] D. H. Moore, J. Donnelly, W. P. McGuire et al., "Limited access trial using amifostine for protection against Cisplatin- and three-hour paclitaxel-induced neurotoxicity: a phase II study of the Gynecologic Oncology Group," Journal of Clinical Oncology, vol. 21, no. 22, pp. 4207-4213, 2003.

[45] N. Masuda, S. Negoro, F. Hausheer et al., "Phase I and pharmacologic study of BNP7787, a novel chemoprotector in patients with advanced non-small cell lung cancer," Cancer Chemotherapy and Pharmacology, vol. 67, no. 3, pp. 533-542, 2011.

[46] D. R. Gandara, V. J. Wiebe, E. A. Perez, R. W. Makuch, and M. W. DeGregorio, "Cisplatin rescue therapy: experience with sodium thiosulfate, WR2721, and diethyldithiocarbamate," Critical Reviews in Oncology/Hematology, vol. 10, no. 4, pp. 353-365, 1990.

[47] G. Tredici, M. Braga, G. Nicolini et al., "Effect of recombinant human nerve growth factor on Cisplatin neurotoxicity in rats," Experimental Neurology, vol. 159, no. 2, pp. 551-558, 1999.
[48] L. Aloe, L. Manni, F. Properzi, S. de Santis, and M. Fiore, "Evidence that nerve growth factor promotes the recovery of peripheral neuropathy induced in mice by Cisplatin: behavioral, structural and biochemical analysis," Autonomic Neuroscience, vol. 86, no. 1-2, pp. 84-93, 2000.

[49] W. Q. Gao, N. Dybdal, N. Shinsky et al., "Neurotrophin3 reverses experimental Cisplatin-induced peripheral sensory neuropathy," Annals of Neurology, vol. 38, no. 1, pp. 30-37, 1995.

[50] I. D. Davis, L. Kiers, L. MacGregor et al., "A randomized, double-blinded, placebo-controlled phase II trial of recombinant human leukemia inhibitory factor (rhuLIF, Emfilermin, AM424) to prevent chemotherapy-induced peripheral neuropathy," Clinical Cancer Research, vol. 11, no. 5, pp. 1890-1898, 2005.

[51] I. Cervellini, E. Bello, R. Frapolli et al., "The neuroprotective effect of erythropoietin in docetaxel-induced peripheral neuropathy causes no reduction of antitumor activity in 13,762 adenocarcinoma-bearing rats," Neurotoxicity Research, vol. 18, no. 2, pp. 151-160, 2010.

[52] R. Bianchi, A. Gilardini, V. Rodriguez-Menendez et al., "Cisplatin-induced peripheral neuropathy: neuroprotection by erythropoietin without affecting tumour growth," European Journal of Cancer, vol. 43, no. 4, pp. 710-717, 2007.

[53] M. Dicato and L. Plawny, "Erythropoietin in cancer patients: pros and cons," Current Opinion in Oncology, vol. 22, no. 4, pp. 307-311, 2010.

[54] S. Cascinu, L. Cordella, E. del Ferro, M. Fronzoni, and G. Catalano, "Neuroprotective effect of reduced glutathione on Cisplatin-based chemotherapy in advanced gastric cancer: a randomized double-blind placebo- controlled trial," Journal of Clinical Oncology, vol. 13, no. 1, pp. 26-32, 1995.

[55] S. Cascinu, V. Catalano, L. Cordella et al., "Neuroprotective effect of reduced glutathione on oxaliplatin-based chemotherapy in advanced colorectal cancer: a randomized, double-blind, placebo-controlled trial," Journal of Clinical Oncology, vol. 20, no. 16, pp. 3478-3483, 2002.

[56] L. Bove, M. Picardo, V. Maresca, B. Jandolo, and A. Pace, "A pilot study on the relation between Cisplatin neuropathy and vitamin E," Journal of Experimental and Clinical Cancer Research, vol. 20, no. 2, pp. 277-280, 2001.

[57] A. A. Argyriou, E. Chroni, A. Koutras et al., "Vitamin E for prophylaxis against chemotherapy-induced neuropathy: a randomized controlled trial," Neurology, vol. 64, no. 1, pp. 26-31, 2005.

[58] A. A. Argyriou, E. Chroni, A. Koutras et al., "Preventing paclitaxel-induced peripheral neuropathy: a phase II trial of vitamin E supplementation," Journal of Pain and Symptom Management, vol. 32, no. 3, pp. 237-244, 2006.

[59] A. Pace, D. Giannarelli, E. Galiè et al., "Vitamin E neuroprotection for Cisplatin neuropathy: a randomized, placebocontrolled trial," Neurology, vol. 74, no. 9, pp. 762-766, 2010.

[60] C. Pisano, G. Pratesi, D. Laccabue et al., "Paclitaxel and Cisplatin-induced neurotoxicity: a protective role of acetyl-Lcarnitine," Clinical Cancer Research, vol. 9, no. 15, pp. 5756-5767, 2003.

[61] W. Boehmerle, K. Zhang, M. Sivula et al., "Chronic exposure to paclitaxel diminishes phosphoinositide signaling by calpainmediated neuronal calcium sensor-1 degradation," Proceedings of the National Academy of Sciences of the United States of America, vol. 104, no. 26, pp. 11103-11108, 2007. 
[62] V. A. Carozzi, A. Chiorazzi, A. Canta et al., "Glutamate carboxypeptidase inhibition reduces the severity of chemotherapyinduced peripheral neurotoxicity in rat," Neurotoxicity Research, vol. 17, no. 4, pp. 380-391, 2010.

[63] P. C. Kurniali, L. G. Luo, and A. B. Weitberg, "Role of calcium/ magnesium infusion in oxaliplatin-based chemotherapy for colorectal cancer patients," Oncology, vol. 24, no. 3, pp. 289-292, 2010.

[64] G. Cavaletti and P. Marmiroli, "The role of growth factors in the prevention and treatment of chemotherapy-induced peripheral neurotoxicity," Current Drug Safety, vol. 1, no. 1, pp. 35-42, 2006.

[65] J. Albers, V. Chaudhry, G. Cavaletti, and R. Donehower, "Interventions for preventing neuropathy caused by Cisplatin and related compounds," Cochrane Database of Systematic Reviews, vol. 1, Article ID CD005228, 2007.

[66] S. Wolf, D. Barton, L. Kottschade, A. Grothey, and C. Loprinzi, "Chemotherapy-induced peripheral neuropathy: prevention and treatment strategies," European Journal of Cancer, vol. 44, no. 11, pp. 1507-1515, 2008.

[67] G. Kannarkat, E. E. Lasher, and D. Schiff, "Neurologic complications of chemotherapy agents," Current Opinion in Neurology, vol. 20, no. 6, pp. 719-725, 2007.

[68] A. Muthuraman and N. Singh, "Attenuating effect of Acorus calamus extract in chronic constriction injury induced neuropathic pain in rats: an evidence of anti-oxidative, antiinflammatory, neuroprotective and calcium inhibitory effects," BMC Complementary and Alternative Medicine, vol. 11, article 24, 2011.

[69] A. Muthuraman, N. Singh, and A. S. Jaggi, "Effect of hydroalcoholic extract of Acorus calamus on tibial and sural nerve transection-induced painful neuropathy in rats," Journal of Natural Medicines, vol. 65, no. 2, pp. 282-292, 2011.

[70] A. Muthuraman and N. Singh, "Attenuating effect of hydroalcoholic extract of Acorus calamus in vincristine-induced painful neuropathy in rats," Journal of Natural Medicines, vol. 65, no. 34, pp. 480-487, 2011.

[71] E. J. Rahn, A. M. Zvonok, G. A. Thakur, A. D. Khanolkar, A. Makriyannis, and A. G. Hohmann, "Selective activation of cannabinoid $\mathrm{CB}_{2}$ receptors suppresses neuropathic nociception induced by treatment with the chemotherapeutic agent paclitaxel in rats," Journal of Pharmacology and Experimental Therapeutics, vol. 327, no. 2, pp. 584-591, 2008.

[72] R. J. Ellis, W. Toperoff, F. Vaida et al., "Smoked medicinal cannabis for neuropathic pain in HIV: a randomized, crossover clinical trial," Neuropsychopharmacology, vol. 34, no. 3, pp. 672680, 2009.

[73] M. Karst, K. Salim, S. Burstein, I. Conrad, L. Hoy, and U. Schneider, "Analgesic effect of the synthetic cannabinoid CT3 on chronic neuropathic pain: a randomized controlled trial," Journal of the American Medical Association, vol. 290, no. 13, pp. 1757-1762, 2003.

[74] M. A. Ware, T. Wang, S. Shapiro et al., "Smoked cannabis for chronic neuropathic pain: a randomized controlled trial," Canadian Medical Association Journal, vol. 182, no. 14, pp. E694E701, 2010.

[75] D. J. Rog, T. J. Nurmikko, T. Friede, and C. A. Young, "Randomized, controlled trial of cannabis-based medicine in central pain in multiple sclerosis," Neurology, vol. 65, no. 6, pp. 812-819, 2005.

[76] D. R. Blake, P. Robson, M. Ho, R. W. Jubb, and C. S. McCabe, "Preliminary assessment of the efficacy, tolerability and safety of a cannabis-based medicine (Sativex) in the treatment of pain caused by rheumatoid arthritis," Rheumatology, vol. 45, no. 1, pp. 50-52, 2006.

[77] J. S. Berman, C. Symonds, and R. Birch, "Efficacy of two cannabis based medicinal extracts for relief of central neuropathic pain from brachial plexus avulsion: results of a randomised controlled trial," Pain, vol. 112, no. 3, pp. 299-306, 2004.

[78] B. Wilsey, T. Marcotte, A. Tsodikov et al., "A randomized, placebo-controlled, crossover trial of cannabis cigarettes in neuropathic pain," The Journal of Pain, vol. 9, no. 6, pp. 506-521, 2008.

[79] D. I. Abrams, C. A. Jay, S. B. Shade et al., "Cannabis in painful HIV-associated sensory neuropathy: a randomized placebocontrolled trial," Neurology, vol. 68, no. 7, pp. 515-521, 2007.

[80] R. J. Ellis, W. Toperoff, F. Vaida et al., "Smoked medicinal cannabis for neuropathic pain in HIV: a randomized, crossover clinical trial," Neuropsychopharmacology, vol. 34, no. 3, pp. 672680, 2009.

[81] J. S. Berman, C. Symonds, and R. Birch, "Efficacy of two cannabis based medicinal extracts for relief of central neuropathic pain from brachial plexus avulsion: results of a randomised controlled trial," Pain, vol. 112, no. 3, pp. 299-306, 2004.

[82] M. A. Ware, T. Wang, S. Shapiro et al., "Smoked cannabis for chronic neuropathic pain: a randomized controlled trial," Canadian Medical Association Journal, vol. 182, no. 14, pp. E694E701, 2010.

[83] E. T. Varro, R. B. Lynn, and E. R. James, Pharmacognosy, Lea \& Fabigen, Philadelphia, Pa, USA, 9th edition, 1988.

[84] G. B. Norman and W. Max, Herbal Drugs and Phytopharmaceuticals, CRC Press, London, UK, 2nd edition, 2001.

[85] A. N. A. Abad, M. H. K. Nouri, A. Gharjanie, and F. Tavakoli, "Effect of Matricaria chamomilla hydroalcoholic extract on Cisplatin-induced neuropathy in mice," Chinese Journal of Natural Medicines, vol. 9, no. 2, pp. 126-131, 2011.

[86] G. Oztürk, O. Anlar, ErdoğanE, M. Kösem, H. Ozbek, and A. Türker, "The effect of Ginkgo extract EGb761 in Cisplatininduced peripheral neuropathy in mice," Toxicology and Applied Pharmacology, vol. 196, no. 1, pp. 169-175, 2004.

[87] J. Marshall, A. Zakari, J. J. Hwang, V. Papadopoulos, A. Rosenberg, and C. Silver, "Ginkgo biloba, (GB) extract as a neuroprotective agent in oxaliplatin $(\mathrm{Ox})$-induced neuropathy. American Society of Clinical Oncologists Annual Meeting Proceedings," Journal of Clinical Oncology, vol. 22, supplement 14, abstract 3670, 2004.

[88] A. N. A. Abad, M. H. K. Nouri, and F. Tavakkoli, "Effect of Salvia officinalis hydroalcoholic extract on vincristine-induced neuropathy in mice," Chinese Journal of Natural Medicines, vol. 9, no. 5, pp. 354-358, 2011.

[89] J. W. Park, J. H. Jeon, J. W. Yoon et al., "Effects of sweet bee venom pharmacopuncture treatment for chemotherapyinduced peripheral neuropathy: a case series," Integrative Cancer Therapies, vol. 11, no. 2, pp. 166-171, 2011.

[90] J. Yoon, J. H. Jeon, Y. W. Lee et al., "Sweet bee venom pharmacopuncture for chemotherapy-induced peripheral neuropathy," Journal of Acupuncture and Meridian Studies, vol. 5, no. 4, pp. 156-165, 2012.

[91] F. Xu, S. Xu, L. Wang et al., "Antinociceptive efficacy of verticinone in murine models of inflammatory pain and paclitaxel induced neuropathic pain," Biological and Pharmaceutical Bulletin, vol. 34, no. 9, pp. 1377-1382, 2011. 
[92] Y. Y. Sun, Y. J. Jia, M. N. Huang, and J. Chen, "Buyang huanwu decoction in prevention of peripheral neuropathy after chemotherapy: a clinical observation," Guangming Journal of Chinese Medicine, vol. 23, no. 7, pp. 958-959, 2008.

[93] J. H. Deng and S. L. Zou, "Observation on TCM treatment of 32 cases of chemotherapy-induced peripheral neuropathy," Journal of Practical Traditional Chinese Internal Medicine, vol. 21, no. 2, 2007.

[94] L. Pan, H. Gao, and X. R. Xing, "Combined application of traditional Chinese medicine prevention of taxol chemotherapy-induced peripheral neuropathy: a clinical observation," Neimenggu Zhong Yi Yao, vol. 3, p. 28, 2012.

[95] L. Sima and L. Pan, "Influence of Chinese herb on chemotherapy-induced peripheral neuropathy," Annals of Oncology, vol. 20, supplement 3, pp. iii45-iii46, 2009.

[96] K. Hashimoto, Y. Sakuma, and J. Kotani, "Histological study of a paclitaxel-induced peripheral neuropathy model treated with goshajnkigan," The Journal of Osaka Dental University, vol. 38, no. 2, pp. 109-112, 2004.

[97] K. Hashimoto, Y. Sakuma, and J. Kotani, "Goshajinkigan improves paclitaxel-induced peripheral neuropathy in rats," The Journal of Osaka Dental University, vol. 40, no. 1, pp. 47-52, 2006.

[98] S. Ushio, N. Egashira, H. Sada et al., "Goshajinkigan reduces oxaliplatin-induced peripheral neuropathy without affecting anti-tumour efficacy in rodents," European Journal of Cancer, vol. 48, no. 9, pp. 1407-1413, 2012.

[99] Y. Shindo, K. Tenma, H. Imano, M. Hibino, K. Yoshino, and M. Nakamura, "Reduction of oxaliplatin-related neurotoxicity by Gosha-jinki-gan," Gan to Kagaku Ryoho, vol. 35, no. 5, pp. 863865, 2008.

[100] M. Nishioka, M. Shimada, N. Kurita et al., "The Kampo medicine, Goshajinkigan, prevents neuropathy in patients treated by FOLFOX regimen," International Journal of Clinical Oncology, vol. 16, no. 4, pp. 322-327, 2011.

[101] T. Kono, N. Mamiya, N. Chisato et al., "Efficacy of Goshajinkigan for peripheral neurotoxicity of oxaliplatin in patients with advanced or recurrent colorectal cancer," Evidence-Based Complementary and Alternative Medicine, vol. 2011, Article ID 418481, 8 pages, 2011.

[102] T. Yamamoto, T. Murai, M. Ueda et al., "Clinical features of paclitaxel-induced peripheral neuropathy and role of Gosyajinki-gan," Gan to Kagaku Ryoho, vol. 36, no. 1, pp. 89-92, 2009 (Japanese).

[103] H. Kaku, S. Kumagai, H. Onoue et al., "Objective evaluation of the alleviating effects of Goshajinkigan on peripheral neuropathy induced by paclitaxel/carboplatin therapy: a multicenter collaborative study," Experimental and Therapeutic Medicine, vol. 3, no. 1, pp. 60-65, 2012.

[104] T. Yamada, H. Kan, S. Matsumoto et al., "Reduction in oxaliplatin-related neurotoxicity by the administration of Keishikajutsubuto (TJ-18)and powdered processed aconite root," Gan to Kagaku Ryoho, vol. 39, no. 11, pp. 1687-1691, 2012 (Japanese).

[105] T. Tatsumi, D. Kishi, and T. Kogure, “The efficacy of ogikeishigomotsuto on chronic cumulative sensory neuropathy induced by Oxaliplatin-case report and literature view," Journal of Traditional Medicines, vol. 26, no. 3, pp. 136-140, 2009.

[106] T. Hidaka, T. Shima, K. Nagira et al., "Herbal medicine Shakuyaku-kanzo-to reduces paclitaxel-induced painful peripheral neuropathy in mice," European Journal of Pain, vol. 13, no. 1, pp. 22-27, 2009.
[107] K. Fujii, S. Okamoto, K. Saitoh et al., "The efficacy of ShakuyakuKanzo-to for peripheral nerve dysfunction in paclitaxel combination chemotherapy for epithelial ovarian carcinoma," Gan to Kagaku Ryoho, vol. 31, no. 10, pp. 1537-1540, 2004.

[108] A. Hosokawa, K. Ogawa, T. Ando et al., "Preventive effect of traditional Japanese medicine on neurotoxicity of FOLFOX for metastatic colorectal cancer: a multicenter retrospective study," Anticancer Research, vol. 32, no. 7, pp. 2545-2550, 2012.

[109] F. V. Defeudis, "Bilobalide and neuroprotection," Pharmacological Research, vol. 46, no. 6, pp. 565-568, 2002.

[110] F. V. DeFeudis, V. Papadopoulos, and K. Drieu, "Ginkgo biloba extracts and cancer: a research area in its infancy," Fundamental and Clinical Pharmacology, vol. 17, no. 4, pp. 405-417, 2003.

[111] B. Ahlemeyer and J. Krieglstein, "Pharmacological studies supporting the therapeutic use of Ginkgo biloba extract for Alzheimer's disease," Pharmacopsychiatry, vol. 36, supplement 1, pp. 8-14, 2003.

[112] H. J. P. Dorman, S. G. Deans, R. C. Noble et al., "Evaluation in vitro of plant essential oils as natural antioxidants," Journal of Essential Oil Research, vol. 7, no. 6, pp. 645-651, 1995.

[113] J. Hohmann, I. Zupkó, D. Rédei et al., "Protective effects of the aerial parts of Salvia officinalis, Melissa officinalis and Lavandula angustifolia and their constituents against enzyme-dependent and enzyme-independent lipid peroxidation," Planta Medica, vol. 65 , no. 6, pp. 576-578, 1999.

[114] D. Malencić, O. Gasic, M. Popović, and P. Boza, "Screening for antioxidant properties of Salvia reflexa hornem," Phytotherapy Research, vol. 14, no. 7, pp. 546-548, 2000.

[115] I. Zupkó, J. Hohmann, D. Rédei, G. Falkay, G. Janicsák, and I. Máthé, "Antioxidant activity of leaves of Salvia species in enzyme-dependent and enzyme-independent systems of lipid peroxidation and their phenolic constituents," Planta Medica, vol. 67, no. 4, pp. 366-368, 2001.

[116] C. N. Wang, C. W. Chi, Y. L. Lin, C. F. Chen, and Y. J. Shiao, "The neuroprotective effects of phytoestrogens on amyloid $\beta$ proteininduced toxicity are mediated by abrogating the activation of caspase cascade in rat cortical neurons," The Journal of Biological Chemistry, vol. 276, no. 7, pp. 5287-5295, 2001.

[117] M. J. Howes, N. S. Perry, and P. J. Houghton, "Plants with traditional uses and activities, relevant to the management of Alzheimer's disease and other cognitive disorders," Phytotherapy Research, vol. 17, no. 1, pp. 1-18, 2003.

[118] Y. A. Maklad, E. A. Aboutabl, M. M. El-Sherei, and K. M. Meselhy, "Bioactivity studies of Salvia transsylvanica (Schur ex Griseb) grown in Egypt," Phytotherapy Research, vol. 13, no. 2, pp. 147-150, 1999.

[119] M. R. A. Rodrigues, L. K. S. Kanazawa, T. L. M. D. Neves et al., "Antinociceptive and anti-inflammatory potential of extract and isolated compounds from the leaves of Salvia officinalis in mice," Journal of Ethnopharmacology, vol. 139, no. 2, pp. 519-526, 2012.

[120] S. Y. Yoon, D. H. Roh, Y. B. Kwon et al., "Acupoint stimulation with diluted bee venom (apipuncture) potentiates the analgesic effect of intrathecal clonidine in the rodent formalin test and in a neuropathic pain model," The Journal of Pain, vol. 10, no. 3, pp. 253-263, 2009.

[121] S. Y. Kang, D. H. Roh, S. Y. Yoon et al., "Repetitive treatment with diluted bee venom reduces neuropathic pain via potentiation of locus coeruleus noradrenergic neuronal activity and modulation of spinal NR1 phosphorylation in rats," The Journal of Pain, vol. 13, no. 2, pp. 155-166, 2012. 
[122] Q. R. Wang, Yi Lin Gai Cuo: Correcting the Errors in the Forest of Medicine, Blue Poppy Press, Boulder, Colo, USA, 2007.

[123] Y. Qian, Xiaoer Yaozheng Zhijue. With coments by X. Z. Yan, J. S. Guo. People's Medical Publishing House. 2006.

[124] Z. J. Zhang, On Cold Damage (Shang Han Lun), Translation \& Commentaries by Y. Feng, N. Wiseman, C. Mitchell and Y. Feng, Blue Poppy Press, Boulder, Colo, USA, 2000.

[125] Y. H. Yan, Jisheng fang, first published in 1253. Reprint Shangwu Yin Shu Guan, Taibei, Taiwan, 1975.

[126] Japan Society for Oriental Medicine, Introduction to Kampo. Japanese Traditional Medicine, Elsevier, Tokio, Japan, 2005.

[127] Y. Niwa and Y. Miyachi, "Antioxidant action of natural health products and Chinese herbs," Inflammation, vol. 10, no. 1, pp. 79-91, 1986.

[128] B. J. Kim, J. H. Kim, H. P. Kim, and M. Y. Heo, "Biological screening of 100 plant extracts for cosmetic use (II): antioxidative activity and free radical scavenging activity, International Journal of Cosmetic Science, vol. 19, no. 6, pp. 299-307, 1997.

[129] M. Tawata, A. Kurihara, K. Nitta, E. Iwase, N. Gan, and T. Onaya, "The effects of Goshajinkigan, a herbal medicine, on subjective symptoms and vibratory threshold in patients with diabetic neuropathy," Diabetes Research and Clinical Practice, vol. 26, no. 2, pp. 121-128, 1994.

[130] T. Kono, H. Mishima, M. Shimada, S. Morita, and J. Sakamoto, "Preventive effect of Goshajinkigan on peripheral neurotoxicity of FOLFOX therapy: a placebo-controlled double-blind randomized phase II study (the GONE Study)," Japanese Journal of Clinical Oncology, vol. 39, no. 12, pp. 847-849, 2009.

[131] M. Nakanishi, J. Arimitsu, M. Kageyama et al., "Efficacy of traditional Japanese herbal medicines-Keishikajutsubuto (TJ18) and Bushi-matsu (TJ-3022)-against postherpetic neuralgia aggravated by self-reported cold stimulation: a case series," The Journal of Alternative and Complementary Medicine, vol. 18, no. 7, pp. 686-692, 2012.

[132] J. Z. Zhang, Gui Yao Lue (Essential Prescriptions from the Golden Chamber), Translated by Nigel Wiseman, Paradigm Publications, Taos, NM, USA, 2013.

[133] H. Hikiami, K. Yagi, S. Nakata et al., "Two cases of numbness and pain of neuropathy due to ANCA-associated vasculitis successfully treated with ogikeishigomotsuto," Kampo Medicine, vol. 58, no. 3, pp. 495-501, 2007.

[134] H. Fujiwara, T. Urabe, K. Ueda et al., "Prevention of arthralgia and myalgia from paclitaxel and carboplatin combination chemotherapy with Shakuyaku-kanzo-to," Gan to Kagaku Ryoho, vol. 27, no. 7, pp. 1061-1064, 2000.

[135] K. Hasegawa, Y. Mizutani, H. Kuramoto et al., "The effect of L-Glutamine and Shakuyaku-Kanzo-to for paclitaxel-induced myalgia/arthralgia," Gan to Kagaku Ryoho, vol. 29, no. 4, pp. 569-574, 2002.

[136] K. Yamamoto, H. Hoshiai, and K. Noda, "Effects of Shakuyakukanzo-to on muscle pain from combination chemotherapy with paclitaxel and carboplatin," Gynecologic Oncology, vol. 81, no. 2, pp. 333-334, 2001.

[137] S. Derry, A. Sven-Rice, P. Cole et al., "Topical capsaicin (high concentration) for chronic neuropathic pain in adults," Cochrane Database of Systematic Reviews, vol. 2, Article ID CD007393, 2013.

[138] K. Shibata, T. Sugawara, K. Fujishita et al., "The astrocytetargeted therapy by Bushi for the neuropathic pain in mice," PLoS ONE, vol. 6, no. 8, Article ID e23510, 2011.
[139] H. Xu, H. Arita, M. Hayashida, L. Zhang, H. Sekiyama, and K. Hanaoka, "Pain-relieving effects of processed Aconiti tuber in CCI-neuropathic rats," Journal of Ethnopharmacology, vol. 103, no. 3, pp. 392-397, 2006.

[140] S. Tatsumi, T. Mabuchi, T. Abe, L. Xu, T. Minami, and S. Ito, "Analgesic effect of extracts of Chinese medicinal herbs Moutan cortex and Coicis semen on neuropathic pain in mice," Neuroscience Letters, vol. 370, no. 2-3, pp. 130-134, 2004.

[141] M. Kanter, "Effects of Nigella sativa and its major constituent, thymoquinone on sciatic nerves in experimental diabetic neuropathy," Neurochemical Research, vol. 33, no. 1, pp. 87-96, 2008.

[142] A. Muthuraman, V. Diwan, A. S. Jaggi, N. Singh, and D. Singh, "Ameliorative effects of Ocimum sanctum in sciatic nerve transection-induced neuropathy in rats," Journal of Ethnopharmacology, vol. 120, no. 1, pp. 56-62, 2008.

[143] T. Baluchnejadmojarad, M. Roghani, and F. Roghani-Dehkordi, "Antinociceptive effect of Teucrium polium leaf extract in the diabetic rat formalin test," Journal of Ethnopharmacology, vol. 97, no. 2, pp. 207-210, 2005.

[144] C. A. L. Kassuya, A. A. Silvestre, V. L. G. Rehder, and J. B. Calixto, "Anti-allodynic and anti-oedematogenic properties of the extract and lignans from Phyllanthus amarus in models of persistent inflammatory and neuropathic pain," European Journal of Pharmacology, vol. 478, no. 2-3, pp. 145-153, 2003.

[145] R. B. Pandhare, B. Sangameswaran, P. B. Mohite et al., "Attenuating effect of Sesbania sesban (L) Merr. extract on neuropathic pain in streptozotocin-induced diabetic rats: an evidence of neuroprotective effects," Phytopharmacology, vol. 2, no. 1, pp. 190-201, 2012.

[146] D. M. Eisenberg, E. S. J. Harris, B. A. Littlefield et al., "Developing a library of authenticated Traditional Chinese Medicinal (TCM) plants for systematic biological evaluation-rationale, methods and preliminary results from a Sino-American collaboration," Fitoterapia, vol. 82, no. 1, pp. 17-33, 2011.

[147] Q. Luo, Y. Cai, J. Yan, M. Sun, and H. Corke, "Hypoglycemic and hypolipidemic effects and antioxidant activity of fruit extracts from Lycium barbarum," Life Sciences, vol. 76, no. 2, pp. 137-149, 2004.

[148] Y. Suzuki, K. Goto, A. Ishige, Y. Komatsu, and J. Kamei, "Antinociceptive effect of Gosha-jinki-gan, a Kampo medicine, in streptozotocin-induced diabetic mice," The Japanese Journal of Pharmacology, vol. 79, no. 2, pp. 169-175, 1999.

[149] I. Raskin and C. Ripoll, "Can an apple a day keep the doctor away?" Current Pharmaceutical Design, vol. 10, no. 27, pp. 34193429, 2004.

[150] B. M. Schmidt, D. M. Ribnicky, P. E. Lipsky, and I. Raskin, "Revisiting the ancient concept of botanical therapeutics," Nature Chemical Biology, vol. 3, no. 7, pp. 360-366, 2007.

[151] H. Shu, H. Arita, M. Hayashida, H. Sekiyama, and K. Hanaoka, "Effects of processed Aconiti tuber and its ingredient alkaloids on the development of antinociceptive tolerance to morphine," Journal of Ethnopharmacology, vol. 103, no. 3, pp. 398-405, 2006.

[152] M. Murayama, T. Mori, H. Bando, and T. Amiya, "Studies on the constituents of Aconitum species. IX. The pharmacological properties of pyro-type aconitine alkaloids, components of processed aconite powder 'kako-bushi-matsu': analgesic, antiinflammatory and acute toxic activities," Journal of Ethnopharmacology, vol. 35, no. 2, pp. 159-164, 1991.

[153] K. Yamada, E. Suzuki, T. Nakaki, S. Watanabe, and S. Kanba, "Aconiti tuber increases plasma nitrite and nitrate levels in 
humans," Journal of Ethnopharmacology, vol. 96, no. 1-2, pp. 165-169, 2005.

[154] M. Mitamura, K. Boussery, S. Horie, T. Murayama, and J. van de Voorde, "Vasorelaxing effect of mesaconitine, an alkaloid from Aconitum japonicum, on rat small gastric artery: possible involvement of endothelium-derived hyperpolarizing factor," The Japanese Journal of Pharmacology, vol. 89, no. 4, pp. 380387, 2002.

[155] Y. Suzuki, K. Goto, A. Ishige, Y. Komatsu, and J. Kamei, "Effects of Gosha-jinki-gan, a Kampo medicine, on peripheral tissue blood flow in streptozotocin-induced diabetic rats," Methods and Findings in Experimental and Clinical Pharmacology, vol. 20, no. 4, pp. 321-328, 1998.

[156] Y. Cui, Z. Yan, S. Hou, and Z. Chang, "Effect of radix Rehmanniae preparata on the expression of c-fos and NGF in hippocampi and learning and memory in rats with damaged thalamic arcuate nucleus," Zhong Yao Cai, vol. 27, no. 8, pp. 589$592,2004$.

[157] J. G. Choi, M. Moon, H. U. Jeong, M. C. Kim, S. Y. Kim, and M. S. Oh, "Cistanches Herba enhances learning and memory by inducing nerve growth factor," Behavioural Brain Research, vol. 216, no. 2, pp. 652-658, 2011.

[158] C. Matsumoto, T. Kojima, K. Ogawa et al., "A proteomic approach for the diagnosis of 'Oketsu' (blood stasis), a pathophysiologic concept of Japanese traditional (Kampo) medicine," Evidence-Based Complementary and Alternative Medicine, vol. 5, no. 4, pp. 463-474, 2008.

[159] C. R. Li, Z. Zhou, D. Zhu, Y. N. Sun, J. M. Dai, and S. Q. Wang, "Protective effect of paeoniflorin on irradiation-induced cell damage involved in modulation of reactive oxygen species and the mitogen-activated protein kinases," International Journal of Biochemistry and Cell Biology, vol. 39, no. 2, pp. 426-438, 2007.

[160] Y. Zhu, S. Dang, and Z. Hua, "Advanced achievements about neuroprotective mechanisms of paeoniflorin," Zhongguo Zhong Yao Za Zhi, vol. 35, no. 11, pp. 1490-1493, 2010.

[161] H. Chunnian, P. Yong, F. Yuxiong, P. Bing, W. Zhe, and X. Peigen, "Quick comparison of Radix Paeonia Alba, Radix Paeonia Rubra, and Cortex Moutan by high performance liquid chromatography coupled with monolithic columns and their chemical pattern recognition," Pharmacognosy Magazine, vol. 8, no. 31, pp. 237-243, 2012.

[162] M. C. Lu, C. H. Yao, S. H. Wang, Y. L. Lai, C. C. Tsai, and Y. S. Chen, "Effect of Astragalus membranaceus in rats on peripheral nerve regeneration: in vitro and in vivo studies," Journal of Trauma, vol. 68, no. 2, pp. 434-440, 2010.

[163] J. Yu, Y. Zhang, S. Sun et al., "Inhibitory effects of astragaloside IV on diabetic peripheral neuropathy in rats," Canadian Journal of Physiology and Pharmacology, vol. 84, no. 6, pp. 579-587, 2006.

[164] N. Y. Tang, C. H. Liu, S. Y. Su et al., "Uncaria rhynchophylla (Miq) Jack plays a role in neuronal protection in kainic acidtreated rats," American Journal of Chinese Medicine, vol. 38, no. 2, pp. 251-263, 2010.

[165] H. Y. Zhang, Y. H. Liu, H. Q. Wang, J. H. Xu, and H. T. Hu, "Puerarin protects PC12 cells against $\beta$-amyloid-induced cell injury," Cell Biology International, vol. 32, no. 10, pp. 1230-1237, 2008.

[166] H. Zhang, Y. Liu, M. Lao, Z. Ma, and X. Yi, "Puerarin protects Alzheimer's disease neuronal cybrids from oxidant-stress induced apoptosis by inhibiting pro-death signaling pathways," Experimental Gerontology, vol. 46, no. 1, pp. 30-37, 2011.
[167] D. Sha, L. Li, L. Ye, R. Liu, and Y. Xu, "Icariin inhibits neurotoxicity of $\beta$-amyloid by upregulating cocaine-regulated and amphetamine-regulated transcripts," Neuroreport, vol. 20, no. 17, pp. 1564-1567, 2009.

[168] Y. S. Ho, M. S. Yu, S. Y. Yik, K. F. So, W. H. Yuen, and R. C. Chang, "Polysaccharides from wolfberry antagonizes glutamate excitotoxicity in rat cortical neurons," Cellular and Molecular Neurobiology, vol. 29, no. 8, pp. 1233-1244, 2009.

[169] Y. H. Wang and G. H. Du, "Ginsenoside Rg1 inhibits $\beta$-secretase activity in vitro and protects against $\mathrm{A} \beta$-induced cytotoxicity in PC12 cells," Journal of Asian Natural Products Research, vol. 11, no. 7, pp. 604-612, 2009.

[170] C. P. Hoi, Y. P. Ho, L. Baum, and A. H. L. Chow, "Neuroprotective effect of honokiol and magnolol, compounds from Magnolia officinalis, on beta-amyloid-induced toxicity in PC12 cells," Phytotherapy Research, vol. 24, no. 10, pp. 1538-1542, 2010.

[171] H. W. Zhao and X. Y. Li, "Ginkgolide A, B, and huperzine A inhibit nitric oxide-induced neurotoxicity," International Immunopharmacology, vol. 2, no. 11, pp. 1551-1556, 2002.

[172] E. H. Kim, M. H. Jang, M. C. Shin, M. S. Shin, and C. J. Kim, "Protective effect of aqueous extract of Ginseng radix against 1methyl-4-phenylpyridinium-induced apoptosis in PC12 cells," Biological and Pharmaceutical Bulletin, vol. 26, no. 12, pp. 1668$1673,2003$.

[173] R. C. Choi, Z. Jiang, H. Q. Xie et al., "Anti-oxidative effects of the biennial flower of Panax notoginseng against $\mathrm{H}_{2} \mathrm{O}_{2}$-induced cytotoxicity in cultured PC12 cells," Chinese Medicine, vol. 5, article 38, 2010.

[174] X. Li, K. Matsumoto, Y. Murakami, Y. Tezuka, Y. Wu, and S. Kadota, "Neuroprotective effects of Polygonum multiflorum on nigrostriatal dopaminergic degeneration induced by paraquat and maneb in mice," Pharmacology Biochemistry and Behavior, vol. 82, no. 2, pp. 345-352, 2005.

[175] W. Wang and D. Q. Wang, "Progress of study on brain protective effect and mechanism of Polygonum multiflorum," Zhongguo Zhong Xi Yi Jie He Za Zhi, vol. 25, no. 10, pp. 955-959, 2005.

[176] X. Li, Y. Li, J. Chen et al., "Tetrahydroxystilbene glucoside attenuates $\mathrm{MPP}^{+}$-induced apoptosis in PC12 cells by inhibiting ROS generation and modulating JNK activation," Neuroscience Letters, vol. 483, no. 1, pp. 1-5, 2010.

[177] K. Faust, S. Gehrke, Y. Yang, L. Yang, M. F. Beal, and B. $\mathrm{Lu}$, "Neuroprotective effects of compounds with antioxidant and anti-inflammatory properties in a Drosophila model of Parkinson's disease," BMC Neuroscience, vol. 10, article 109, 2009.

[178] L. L. Tian, X. J. Wang, Y. N. Sun et al., "Salvianolic acid B, an antioxidant from Salvia miltiorrhiza, prevents 6hydroxydopamine induced apoptosis in SH-SY5Y cells," International Journal of Biochemistry and Cell Biology, vol. 40, no. 3, pp. 409-422, 2008.

[179] S. X. Wang, L. M. Hu, X. M. Gao, H. Guo, and G. W. Fan, "Antiinflammatory activity of salvianolic acid B in microglia contributes to its neuroprotective effect," Neurochemical Research, vol. 35, no. 7, pp. 1029-1037, 2010.

[180] W. J. Xia, M. Yang, T. F. Fok et al., "Partial neuroprotective effect of pretreatment with tanshinone IIA on neonatal hypoxiaischemia brain damage," Pediatric Research, vol. 58, no. 4, pp. 784-790, 2005.

[181] E. J. Shin, J. H. Bach, T. T. L. Nguyen et al., "Gastrodia elata bl attenuates methamphetamine-induced dopaminergic toxicity 
via inhibiting oxidative burdens," Current Neuropharmacology, vol. 9, no. 1, pp. 118-121, 2011.

[182] C. L. Hsieh, C. L. Chen, N. Y. Tang et al., "Gastrodia elata BL mediates the suppression of nnos and microglia activation to protect against neuronal damage in kainic acid-treated rats," American Journal of Chinese Medicine, vol. 33, no. 4, pp. 599611, 2005.

[183] S. Y. Li, Y. H. Jia, W. G. Sun et al., "Stabilization of mitochondrial function by tetramethylpyrazine protects against kainateinduced oxidative lesions in the rat hippocampus," Free Radical Biology and Medicine, vol. 48, no. 4, pp. 597-608, 2010.

[184] L. H. Fan, K. Z. Wang, B. Cheng, C. S. Wang, and X. Q. Dang, "Anti-apoptotic and neuroprotective effects of Tetramethylpyrazine following spinal cord ischemia in rabbits," $B M C$ Neuroscience, vol. 7, article 48, 2006.

[185] J. H. Park, H. J. Lee, S. B. Koh, J. Y. Ban, and Y. H. Seong, "Protection of NMDA-induced neuronal cell damage by methanol extract of Zizyphi Spinosi Semen in cultured rat cerebellar granule cells," Journal of Ethnopharmacology, vol. 95, no. 1, pp. 39-45, 2004.

[186] F. Q. Li, T. Wang, Z. Pei, B. Liu, and J. S. Hong, "Inhibition of microglial activation by the herbal flavonoid baicalein attenuates inflammation-mediated degeneration of dopaminergic neurons," Journal of Neural Transmission, vol. 112, no. 3, pp. 331347, 2005.

[187] C. Ma, W. Wang, Y. Y. Chen, R. N. Liu, R. F. Wang, and L. J. $\mathrm{Du}$, "Neuroprotective and antioxidant activity of compounds from the aerial parts of Dioscorea opposita," Journal of Natural Products, vol. 68, no. 8, pp. 1259-1261, 2005.

[188] B. Xue, J. Jiao, L. Zhang et al., "Triptolide upregulates NGF synthesis in rat astrocyte cultures," Neurochemical Research, vol. 32, no. 7, pp. 1113-1119, 2007.

[189] H. Y. Zhang and X. C. Tang, "Neuroprotective effects of huperzine A: new therapeutic targets for neurodegenerative disease," Trends in Pharmacological Sciences, vol. 27, no. 12, pp. 619-625, 2006.

[190] M. S. Rafii, S. Walsh, J. T. Little et al., "A phase II trial of huperzine A in mild to moderate Alzheimer disease," Neurology, vol. 76, no. 16, pp. 1389-1394, 2011.

[191] L. Gao, L. Xiang, Y. Luo, G. Wang, J. Li, and J. Qi, “Gentisides $\mathrm{C}-\mathrm{K}$ : nine new neuritogenic compounds from the traditional Chinese medicine Gentiana rigescens Franch," Bioorganic and Medicinal Chemistry, vol. 18, no. 19, pp. 6995-7000, 2010.

[192] Y. Cui, Z. Yan, S. Hou, and Z. Chang, "Effect of radix Rehmanniae preparata on the expression of c-fos and NGF in hippocampi and learning and memory in rats with damaged thalamic arcuate nucleus," Zhong Yao Cai, vol. 27, no. 8, pp. 589$592,2004$.

[193] Y. Zhu, S. Dang, and Z. Hua, "Advanced achievements about neuroprotective mechanisms of paeoniflorin," Zhongguo Zhong Yao Za Zhi, vol. 35, no. 11, pp. 1490-1493, 2010. 


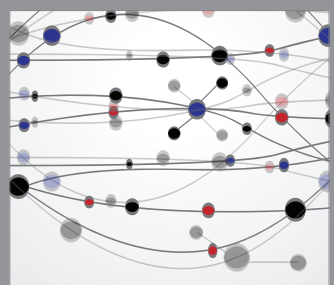

The Scientific World Journal
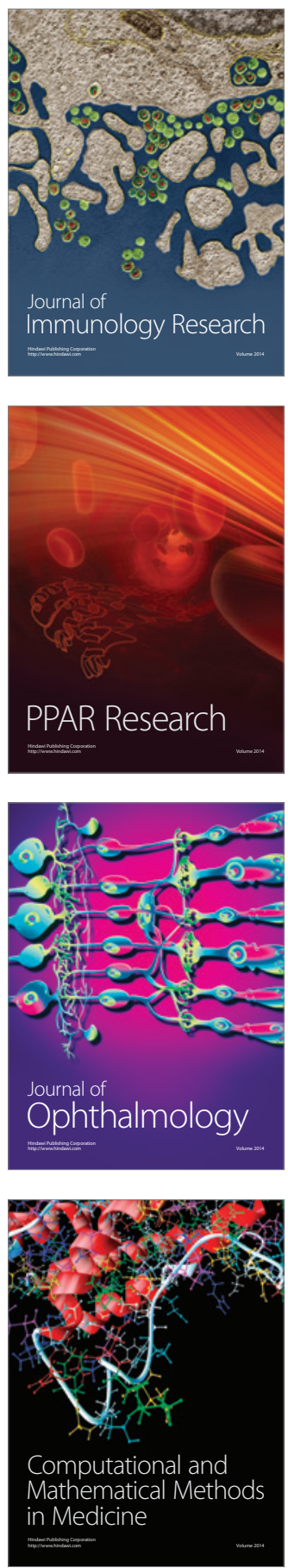

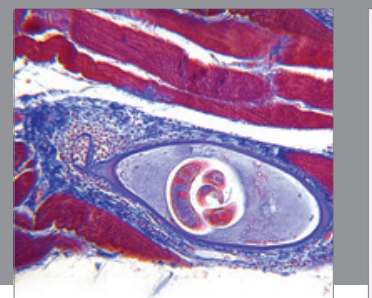

Gastroenterology

Research and Practice
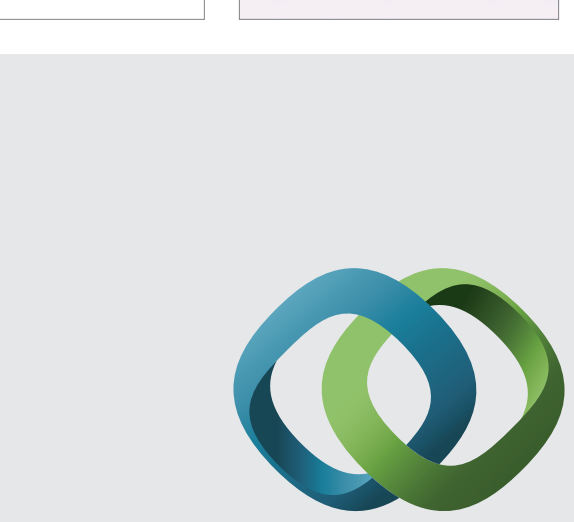

\section{Hindawi}

Submit your manuscripts at

http://www.hindawi.com
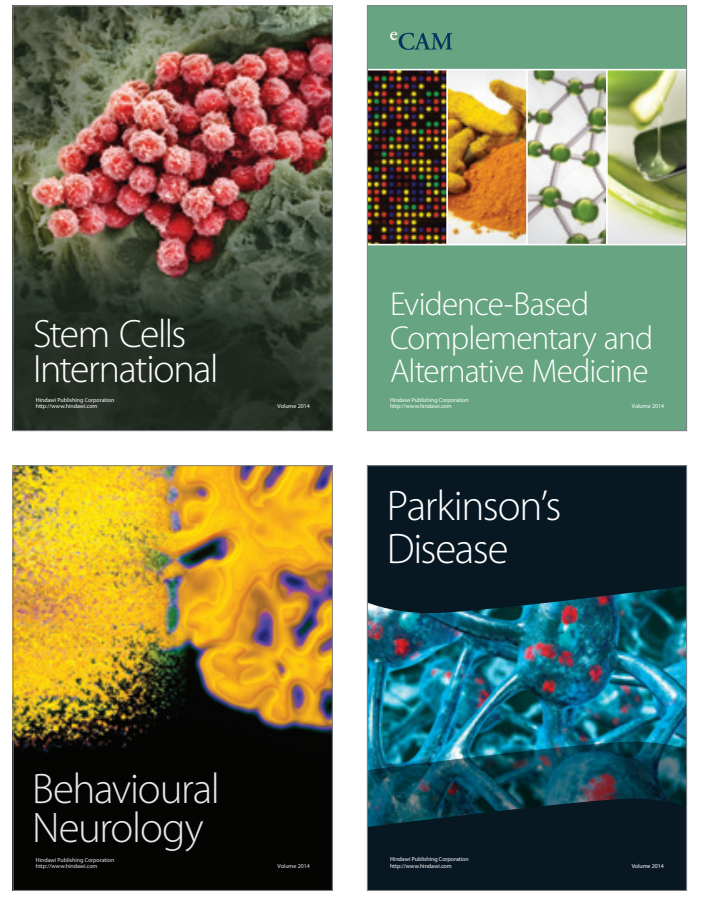
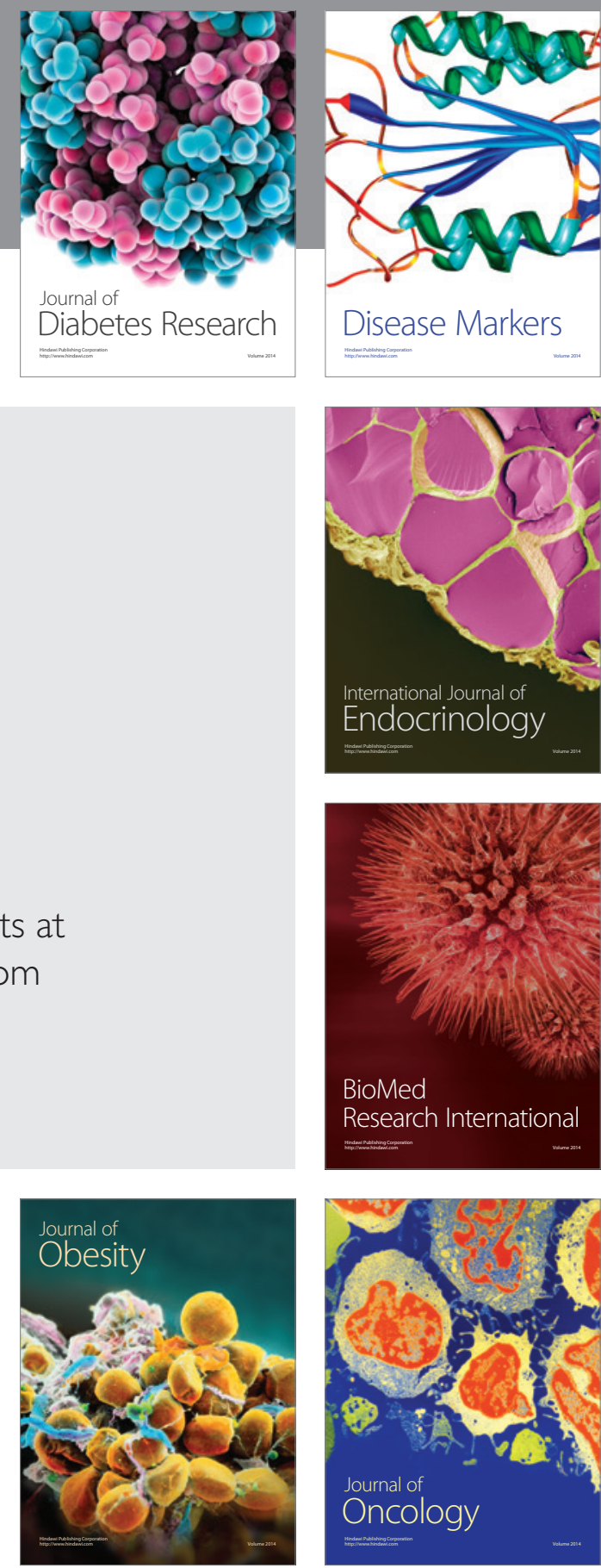

Disease Markers
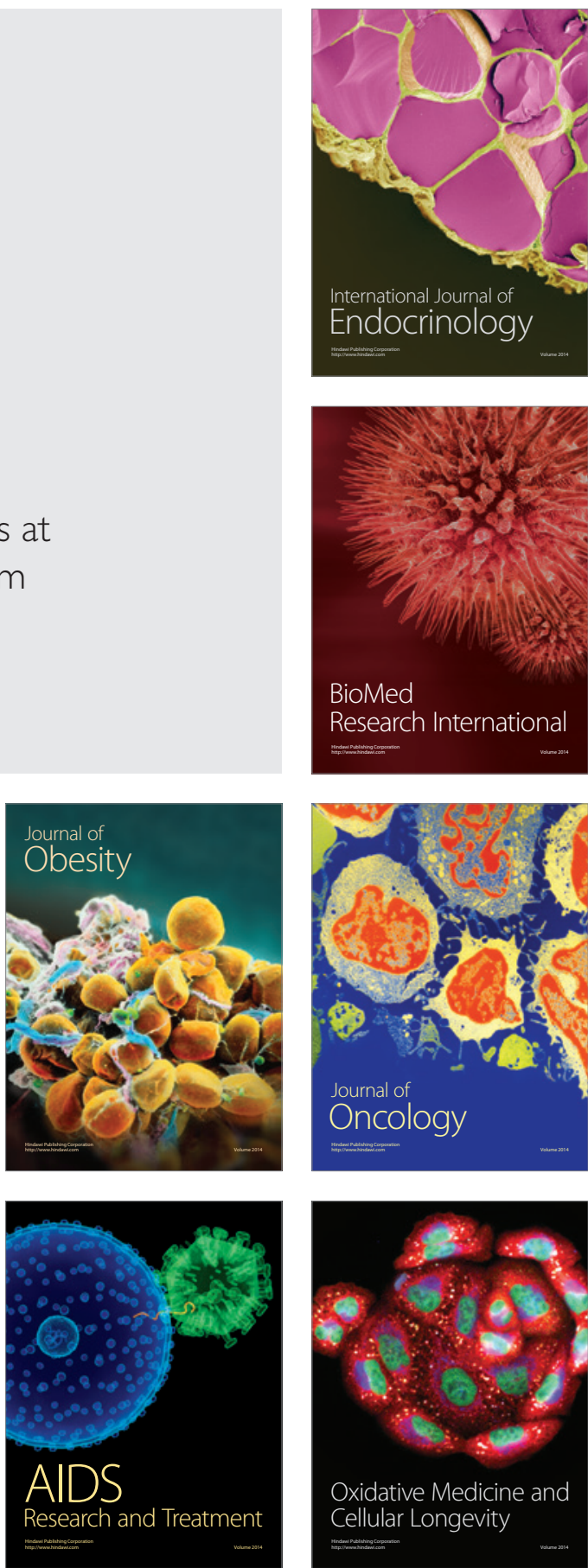